\title{
Chronologie mochica : une nouvelle synthèse
}

Mochica chronology: a new synthesis

Cronología mochica: una nueva síntesis

Nino Del Solar, Rémy Chapoulie et Luis Jaime Castillo

\section{OpenEdition}

\section{Journals}

Édition électronique

URL : https://journals.openedition.org/jsa/14338

DOI : 10.4000/jsa. 14338

ISSN : 1957-7842

\section{Éditeur}

Société des américanistes

Édition imprimée

Date de publication : 31 décembre 2015

Pagination : 233-267

ISSN : 0037-9174

\section{Référence électronique}

Nino Del Solar, Rémy Chapoulie et Luis Jaime Castillo, "Chronologie mochica : une nouvelle synthèse », Journal de la Société des américanistes [En ligne], 101-1 et 2 | 2015, mis en ligne le 15 mars 2016, consulté le 24 février 2023. URL : http://journals.openedition.org/jsa/14338 ; DOI : https:// doi.org/10.4000/jsa.14338 


\title{
Chronologie mochica : une nouvelle synthèse
}

\author{
Nino Del Solar *, Rémy Chapoulie * \\ et Luis Jaime CASTILlO **
}

L'un des enjeux majeurs des sciences archéologiques est l'établissement des séquences chronologiques d'occupation au sein des aires d'activité humaine. L'objectif est la reconstruction des processus culturels diachroniques liés à l'apparition, l'évolution et la fin de cultures matérielles caractéristiques des groupes humains qui ont occupé des espaces environnementaux spécifiques. Concernant la culture mochica ( $\mathrm{II}^{\mathrm{e}}-\mathrm{X}^{\mathrm{e}}$ siècles, côte nord du Pérou), cet objectif est encore au centre de la recherche archéologique et archéométrique contemporaine. Dans cet article, nous proposons une révision et une synthèse des informations concernant la chronologie relative de cette culture. En même temps, nous présentons un inventaire mis à jour de dates ${ }^{14} \mathrm{C}$ mochica obtenues depuis la décennie des années 1970. Il s'agit donc ici de réévaluer les jalons temporels et stylistiques connus et acceptés jusqu'à présent. [Mots-clés: archéologie, Mochica, Pérou, précolombien, datation ${ }^{14} \mathrm{C}$, céramique.]

Mochica chronology: a new synthesis. One of the issues in archaeological sciences is the establishment of chronological sequences in areas of human occupation. The main objective in this context is the reconstruction of the cultural diachronic processes connected to the emergence, the evolution, and the vanishing of the material cultures connected to human societies in specific environments. Concerning the Mochica (2th-10th century, north coast of Peru), recent archaeological research is pursued in order to explain such processes. In this article, we propose a revision and a synthesis of the given information on the relative chronology of this culture. Moreover, we present an inventory of Mochica ${ }^{14} \mathrm{C}$ dates obtained during the last forty years. Thus the goal here is to reassess the temporal and stylistical data accepted by the archaeological community. [Key words: Archaeology, Mochica, Peru, pre-Columbian, $14 \mathrm{C}$ dating, ceramic.]

Cronología mochica: una nueva síntesis. En las ciencias arqueológicas, una de las problemáticas capitales es el establecimiento de secuencias cronológicas de

* Institut de recherche sur les archéomatériaux, UMR 5060 CNRS, Centre de recherche en physique appliquée à l'archéologie (CRP2A), université Bordeaux Montaigne, Maison de l'archéologie, Pessac [nvdelsolarve@u-bordeaux-montaigne.fr; remy.chapoulie@u-bordeauxmontaigne.fr] ** Pontificia Universidad Católica del Perú, Lima, Perú [1castil@pucp.edu.pe]. 
ocupación en áreas de actividad humana. Dicha problemática enmarca como objetivo principal la reconstrucción de los procesos culturales diacrónicos vinculados a la aparición, la evolución y la desaparición de la cultura material característica de un grupo humano establecido en un medio ambiente específico. Para la cultura mochica (siglos II-X d.C., costa norte de Perú), este objetivo es aún central en la investigación arqueológica y arqueométrica contemporánea. En este artículo, proponemos una revisión y una síntesis de las informaciones concernientes a la cronología relativa de la sociedad mochica. Al mismo tiempo, presentamos un inventario actualizado de fechas ${ }^{14} \mathrm{C}$ mochicas obtenidas desde la década de los 1970. El objetivo es reevaluar la información temporal y estilística conocida y aceptada por la comunidad arqueológica. [Palabras clave: arqueología, mochica, Perú, precolombino, datación 14C, cerámica.]

\section{Introduction}

Les sociétés mochica ont occupé la bande côtière ${ }^{1}$ septentrionale de l'actuel territoire péruvien au cours du premier millénaire de notre ère. La culture matérielle mochica a été identifiée sur une aire d'environ $350 \mathrm{~km}$ de long et $60 \mathrm{~km}$ de large. Son influence stylistique couvre un espace global d'environ $550 \mathrm{~km}$ de long entre les vallées de Piura et de Huarmey.

Géographiquement, les sites mochica sont localisés autour d'une dizaine de vallées fluviales (Leche, Lambayeque, Zana, Jequetepeque, Chicama, Moche, Virú, Chao, Santa et Nepeña), dont l'origine est le versant occidental des Andes (Figure 1). Selon leur emplacement, ces vallées sont séparées en deux sections orographiques et hydrographiques qui ont été définies par Weberbauer pour la côte péruvienne $(1980$, p. 15) : la section septentrionale située entre Pisco et Trujillo, et la section localisée entre Trujillo et Punta Pariñas. Ces deux sections composent un vaste territoire caractérisé par la présence de plaines plutôt que de collines, ce qui a toujours permis l'exploitation et la transformation de ce milieu par l'homme : «Cette plaine constitue la plus grande aire agricole de la côte du Pérou [...]. Dans la section entre Trujillo et Punta Pariñas, certains terrains destinés à l'activité agricole arrivent à toucher la mer » (Weberbauer 1980, p. 18 [traduit de l'espagnol]).

Il semble que le développement de systèmes d'irrigation, depuis la période Mochica ancienne, a été à la base de l'accumulation de richesses par les populations aux échelles locales (Billman 2002 ; Castillo et Uceda 2008 ; Castillo 2010). Et c'est aussi cela qui aurait conduit à une complexification sociale (différenciation entre classes productrice et non productrice aux échelles locale et régionale),

1. La côte du Pérou $(\approx 0-0,8 \mathrm{~km}$ d'altitude) est caractérisée par un climat propre aux régions semi-arides sous-tropicales $\left(\approx 18^{\circ} \mathrm{C}\right.$ en moyenne). Géomorphologiquement, elle est composée par des plaines désertiques et des collines peu élevées produites par l'érosion subaérienne et fluviale (Petersen 1980, p. 36 [traduit de l'espagnol]). 
politique (création et planification de cérémonies et d'infrastructures servant à théâtraliser et à légitimer le pouvoir des élites) et économique (enrichissement et accès à la propriété communale) des sociétés côtières.

Concernant l'organisation spatiale du phénomène mochica, la recherche récente a fait évoluer les hypothèses de Rafael Larco (2001) qui concevait l'existence d'un seul territoire, d'une seule entité politique gouvernante et d'une seule culture matérielle largement partagée par une population qui occupait un ensemble de vallées. Aujourd'hui, conformément aux variations que présentent les données archéologiques (ainsi, le phénomène Vicus dans la vallée de Piura), les chercheurs considèrent comme composite et non linéaire l'histoire des Mochica. Probablement issus d'une tradition élitaire post-formative, Gallinazo ou Salinar (Castillo et Uceda 2008), les Mochica auraient suivi des processus culturels particuliers. Ces processus ont été caractérisés par des étapes de centralisation, de décomposition et/ou de conquête au cours des quelque sept siècles de son existence. Cette vision des choses concorde mieux avec les données archéologiques, parfois très hétéroclites. À cause de la discontinuité stylistique des productions matérielles et culturelles mochica entre les vallées du nord et celles du sud, deux séquences céramiques parallèles ont été établies et deux développements culturels reconnus. La présence des deux sphères d'influence culturelle mochica distinctes - dénommées « Mochica nord » et « Mochica sud »-répond mieux aux questionnements liés à l'organisation de l'espace entre la deuxième époque des développements régionaux et l'Horizon moyen (Castillo et Donnan 1994).

En ce qui concerne l'aire Mochica sud (située entre la vallée de Chicama et la vallée de Nepeña), l'hypothèse d'une seule sous-entité politique centralisée (Chapdelaine 2011) et conquérante est encore valable, même si, au cours du temps, des alliances et des divisions ont pu alterner. Les Huacas de Moche auraient été le centre religieux et civique pour cette aire, une sorte de capitale. Il existe d'autres sites monumentaux, dont les rôles sont encore débattus (capitales régionales ou capitales alternatives ?) (Castillo et Uceda 2008).

Dans le cas des Mochica du nord, trois sous-espaces culturels ont été distingués : Mochica de Piura, Mochica de Lambayeque et Mochica de Jequetepeque (Castillo et Uceda 2008; Chapdelaine 2011). Ces trois sous-aires ont été organisées autour des trois systèmes de vallées qui portent les mêmes noms. Mais de nombreuses questions archéologiques se posent : 1. est-ce que la division en trois sous-espaces culturels permet de réfléchir sur l'organisation de la production de la culture matérielle, contrainte par l'environnement et les ressources locales (eau, bois, terres argileuses et minéraux, etc.) ? ; 2. est-ce que des développements aux échelles locales auraient primé sur un développement à une échelle régionale (éventuellement étatique) ?; 3. quels sont les éléments associés au phénomène de la subdivision (en termes d'organisation socio-politique, de mouvements des populations, de disponibilité en matières premières, etc.) ? 

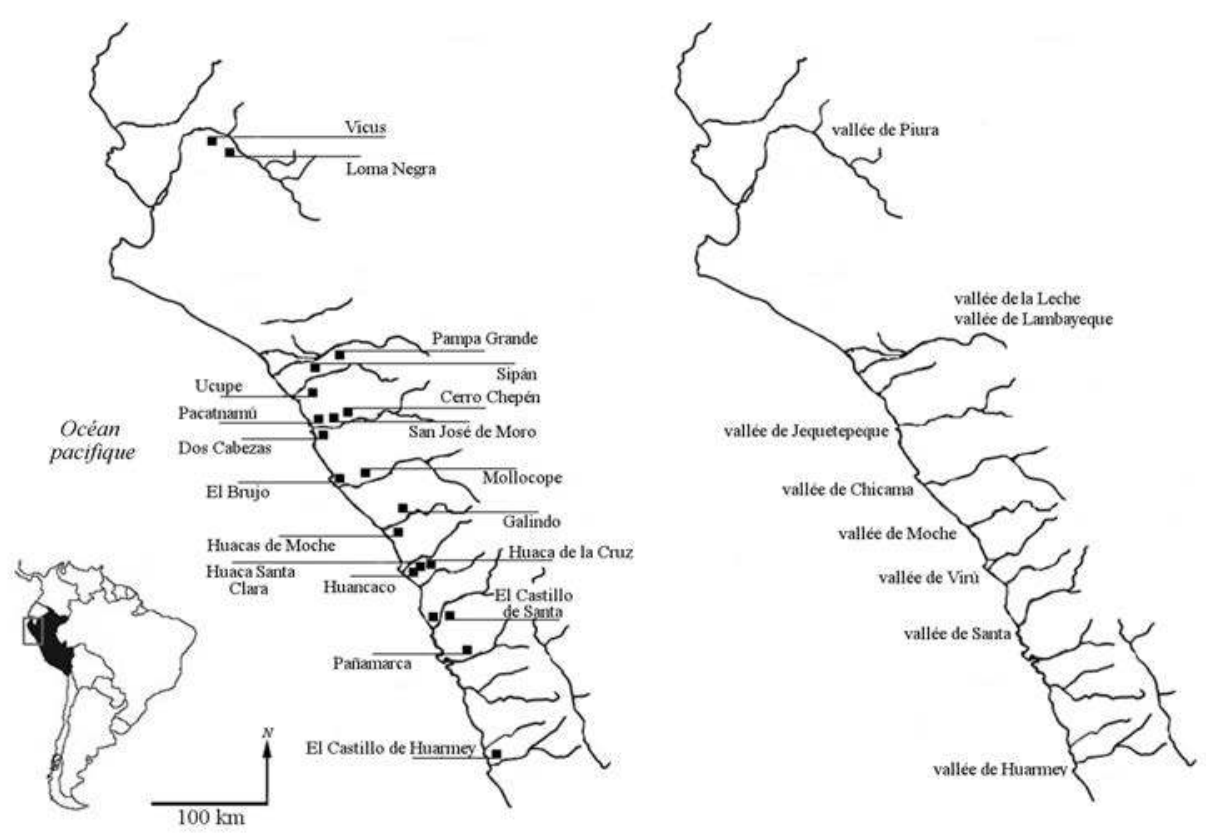

Fig. 1 - Localisation des principaux sites mochica et des vallées sur la côte nord du Pérou.

Légende : — sites archéologiques.

\section{Typo-chronologies mochica}

Le terme « mochica » renvoie à une culture archéologique que l'on date d'entre le II $^{\mathrm{e}}$ et le $\mathrm{x}^{\mathrm{e}}$ siècle apr. J.-C. et qui a pu être définie par plusieurs caractéristiques formelles, spatiales et temporelles (Quilter 2002,p. 152). Les principaux attributs formels des céramiques mochica sont représentés sur l'ensemble du territoire ; toutefois, d'un secteur à l'autre, les séquences typo-céramiques ne sont pas les mêmes. À propos des temporalités des céramiques, la multiplication, au cours des cinquante dernières années, des dates au radiocarbone et des données stylistiques a suscité de nombreuses discussions, notamment sur l'évolution des relations internes et externes des groupes impliqués.

L'existence d'un système d'écriture et d'un calendrier dans les sociétés précolombiennes du Pérou est, encore à ce jour, sujet de débats (voir Larco 1944; Chaparro 2011 [pour la société mochica] ou Urton 2003 ; Beyersdorff 2005 ; Moscovich 2008 [pour les sociétés de l'Horizon tardif]). En l'absence d'inscription de type calendaire et de tout type de date, ce sont la sériation et la stratigraphie qui ont permis d'abord de retracer l'histoire pré-inca et inca (Rowe 1961). Mais, dès la fin des années 1940, plusieurs spécialistes de l'aire 
andine exprimaient la nécessité de trouver des méthodes susceptibles de faire évoluer les mesures relatives en datations absolues. Jusque-là, l'évolution sociale et l'histoire des groupes humains locaux ne s'appuyaient que sur la mise au point de séquences typo-céramiques et des travaux comparatifs. On avait aussi souvent recours aux analogies culturelles, au risque de commettre des anachronismes. Celles-ci cherchent en effet à comparer données archéologiques et ethnographiques sans négliger les données issues de textes historiques des époques coloniale et républicaine.

$\mathrm{Au}$ milieu du $\mathrm{Xx}^{\mathrm{e}}$ siècle, des techniques nouvelles et des approches scientifiques pluridisciplinaires au service des sciences humaines sont apparues. Dans le domaine de l'archéologie, c'est la mesure $d u{ }^{14} \mathrm{C}$ qui a révolutionné le domaine de la datation (Bronk Ramsey 2008) et qui a posé les jalons permettant d'amarrer à l'échelle du temps réel les séquences céramiques précolombiennes (Johnson et al. 1951).

Comme on l'a dit plus haut, les céramiques mochica ont fait l'objet de deux séquences typologiques distinctes, l'une pour la sphère sud (Larco 2001), l'autre pour la sphère nord (Castillo et Donnan 1994). La séquence en cinq phases de Larco Hoyle (I à V) date des années 1950 et elle a été construite grâce au croisement et à la synthèse de différents éléments : la sériation de récipients (bouteilles à anse-étrier, vases-portraits, cancheros, etc.), la caractérisation morphologique des goulots, la décoration, les techniques de fabrication. La plupart des pièces qui ont servi pour la construire provenaient de contextes funéraires. Mais, l'absence de cette typologie au nord (Chapdelaine 2011, p. 195 ; Castillo et Uceda 2008) et des décalages chronologiques et stylistiques la rendaient inadaptée pour caractériser les occupations mochica dans les vallées septentrionales. En réalité, au nord et au sud il y aurait eu des développements différents. La séquence adoptée pour la sphère Mochica nord comprend trois phases : initiale, moyenne et tardive. On remarquera que ces trois phases n'ont jamais été retrouvées toutes les trois sur un même site : c'est le cas par exemple du site de San José de Moro où les occupations connues concernent seulement les phases moyenne et tardive (Castillo 2003). Castillo et Donnan (1994) ont fait une description précise de la typologie de la sphère nord. C'est la période Mochica initiale qui est la moins documentée. Elle n'a été repérée que sur les sites de Tolón, Dos Cabezas et La Mina. Parmi ses caractéristiques les plus remarquables, on note la qualité des sculptures tridimensionnelles, l'abstraction des formes et la similarité entre les anses-étriers des céramiques de cette période et les anses-étriers des pièces de la phase Mochica I au sud. La période Mochica moyenne, quant à elle, a été identifiée surtout sur les sites de Pacatnamú et San José de Moro. Divisées en trois grandes catégories qualitatives (fine, moyenne et simple), les céramiques de cette période présentent des formes variées : bouteilles à anse-étrier, différentes toutefois des pièces du style Mochica III du Sud (forme lenticulaire, goulot modérément évasé, base annulaire, décoration 
en relief, emploi de pigments violets dans la décoration peinte), jarres simples, jarres à col effigie, figurines et marmites à col court, évasé ou droit. Enfin, la phase tardive a été répertoriée sur les sites de Pacatnamú, Chérrepe et San José de Moro. Dans ce dernier gisement, il faut remarquer la découverte de bouteilles à anse-étrier dites de « ligne fine ». Celles-ci comportent des scènes mythologiques assez complexes, des anses en forme de triangle inversé et des goulots coniques. De manière générale, ces bouteilles ont une forte ressemblance avec les bouteilles des phases IV et V de la sériation de Larco Hoyle pour le Sud. À la période tardive du Nord, il est aussi possible de remarquer l'apparition de formes nouvelles (par exemple des jarres mammiformes) et de styles (et/ou de techniques) étrangers (en provenance de Wari, Cajamarca, Lima, Nievería...) dans la production de l'époque. Plus récemment, Donnan (2011) a proposé un nouveau système de classification stylistique des céramiques. Selon lui, les différentes entités politiques au sein du territoire mochica auraient produit des sous-styles différentiables et caractéristiques de chaque entité : ces particularismes auraient visé à exprimer les identités de chacune d'entre elles. Jusqu'à présent, Donnan a défini quatre sous-styles : Moro, Dos Cabezas, Huancaco et Huacas de Moche. À chacun aurait correspondu un centre de production (peut-être sur le site éponyme), ainsi qu'une apparition, une évolution et une fin propres.

\section{Datations mochica au radiocarbone}

La mesure du radiocarbone (Bronk Ramsey 2008) permet de placer les vestiges archéologiques sur l'échelle absolue du temps indépendamment des classifications et des évolutions stylistiques préalablement établies. Elle a pour conséquence la réévaluation d'hypothèses sur les évolutions sociales et stylistiques concernant en l'occurrence les Mochica. Grâce aux dates et aux données issues de fouilles récentes, on constate que les cadres chronologiques et culturels préalablement établis à propos des Mochica sont en train de changer de manière significative (voir Chapdelaine 2011 ; Quilter 2002, p. 151).

À l'échelle de toute l'aire mochica, les premières données ${ }^{14} \mathrm{C}$ remontent aux années 1950 : elles ont été obtenues à partir de matériaux archéologiques des vallées de Moche et de Chicama (Johnson et al. 1951). De manière générale, les datations au radiocarbone ont augmenté fortement depuis les années 1970 et ont été publiées dans les années 1990 et 2000. Uceda et al. (2001 [pour le site de Huacas de Moche]), Lockard (2009 [pour la sphère Mochica sud]) et Koons et Alex (2014 [pour les deux sphères]) ont formulé les premiers bilans sur les datations ${ }^{14} \mathrm{C}$, soit sur un site, soit aux échelles régionale et macrorégionale. Dans la plupart des cas, les datations dont on dispose proviennent de contextes qui comportent des pièces céramiques caractérisables d'un point de vue typologique et stratigraphique. 
Sur la base de l'étude systématique de la littérature relative au phénomène mochica, nous avons recueilli et synthétisé l'information concernant 121 datations ${ }^{14} \mathrm{C}$ (publiées entre 1973 et 2014). La vocation de ce travail est quadruple : 1. accroître le nombre des datations publiées récemment par Koons et Alex (2014), en y ajoutant d'autres données ${ }^{14} \mathrm{C}$ provenant de sites comme Loma Negra, Pacatnamú, Huaca Soledad, Huacas de Moche, San José de Moro et Sipán ; 2. rassembler, organiser et présenter, par site et par région, toutes les datations ${ }^{14} \mathrm{C}$ publiées (articles de revues et ouvrages scientifiques, actes de colloques et rapports de fouilles, en omettant celles qui n'ont fait l'objet que de communications personnelles) ; 3 . discuter de la nature des matériaux datés et des quantités soumises aux analyses ; 4. participer au travail d'inventaire des datations mochica et contribuer, par là, au questionnement sur la validité des séquences typo-chronologiques de chaque vallée ou région, l'information répertoriée étant traitée de façon inclusive, en s'appuyant sur des tests de statistiques descriptives.

\section{Questions méthodologiques}

Elles concernent plusieurs aspects : la nature des échantillons, le choix de la courbe de calibration, l'incertitude des mesures et l'approche statistique. La totalité des échantillons retenus est d'origine terrestre, sauf l'échantillon Beta-108281 provenant du site de Huacas de Moche (voir infra). La totalité des datations a été recalibrée avec la courbe ShCal13 (Hogg et al. 2013), sauf 1'échantillon Beta-08281 qui l'a été avec la courbe Marine13 (voir infra).

Toutes les datations ont été traitées à l'aide du logiciel OxCal v4.2 (Bronk Ramsey 2014). Enfin, pour comparer les données recalibrées, seuls les échantillons liés à une seule typo-chronologie ont été traités statistiquement. En conséquence, les échantillons SMU-833, Beta-121764, Gif-11577, et les échantillons provenant de Licapa et El Brujo, ne font pas partie des comparaisons effectuées.

Les résultats (en dates calendaires) sont donnés à $1 \sigma$ et à $2 \sigma$. La comparaison statistique entre les dates a été réalisée en employant le logiciel PAST v2.17b (Hammer et al. 2001).

\section{Choix de la courbe de calibration}

En ce qui concerne le choix de la courbe de calibration pour l'analyse des 121 dates inventoriées, il faut d'abord rappeler que les différences de concentration en ${ }^{14} \mathrm{C}$ entre les deux hémisphères sont bien connues et qu'elles affectent certaines périodes anciennes (McCormac et al. 2002, p. 641 ; Hogg et al. 2009b ; Ogburn 2012, p. 223). Ce constat a conduit à développer des courbes de calibration particulières et des études statistiques ont permis de corriger les âges des échantillons provenant de l'hémisphère sud (McCormac et al. 2002 [41 \pm 14 ] 
et Hogg et al. 2009a [40 \pm 20$]$ ). Sur les chronologies précolombiennes d'Amérique du Sud, on soulignera que l'étude réalisée par Ogburn (2012, p. 223-225) inclut une discussion assez complète sur le choix de la courbe de calibration à appliquer aux dates ${ }^{14} \mathrm{C}$ inca. Cela dit, il n'existe pas pour le moment d'accord sur la courbe de calibration qu'il faudrait appliquer aux échantillons datés par ${ }^{14} \mathrm{C}$ provenant du Pérou précolombien. Ainsi, Grobman et al. (2012 [pour des échantillons de l'époque précéramique de la vallée de Chicama]), Lockard (2009 [pour des échantillons mochica de la sphère sud]), Koons et Alex (2014 [pour des échantillons mochica et licapa]) et León (2007, p. 35-42 [pour des échantillons paracas de Wari-Cerro Colorado] ; 2011 [pour des échantillons inca provenant des différents gisements]) ont employé la courbe ShCal04 et ShCal13. Mais, Finucane et al. (2007, p. 581 [pour des échantillons wari de la vallée d'Ayacucho]), Unkel et Kromer (2009, p. 235 [pour des échantillons paracas et nasca de la côte sud du Pérou]) ou Marsh (2012, p. 205-206 [pour des échantillons tiwanaku en Bolivie]) utilisent la courbe IntCal (04 ou 09). Pour évaluer les dates ${ }^{14} \mathrm{C}$, Ogburn (2012) a remarqué que le choix de la courbe dépend de plusieurs facteurs, notamment la migration annuelle et la migration à long terme de la zone de convergence intertropicale (ZCIT) par rapport à la position latitudinale du site archéologique où les échantillons ont été récupérés. Elle suggère que « la courbe IntCal est la plus appropriée pour dater des sites situés sur les montagnes andines de l'Équateur jusqu'à la Bolivie septentrionale ; par contre, pour dater des sites situés à des latitudes moyennes, de la Bolivie vers le sud, la courbe SHCal semble être la plus convenable » (Ogburn 2012, p. 224 [traduit de l'anglais]).

Dans la plupart des publications, la principale cause de divergence au moment de choisir l'une des courbes est évidemment la méconnaissance de la position de la ZCIT et de son déplacement historique. L'absence d'études approfondies rend le sujet complexe et difficile à trancher. Selon Haug et al. (2001) et Sachs et al. (2009), la ZCIT a eu tendance à migrer vers le sud lors de l'Holocène. Néanmoins, ce phénomène de migration n'aurait pas été constant. Cette hypothèse a été en partie corroborée par Bird et al. (2011a), puisqu'il propose que la ZCIT s'est déplacée vers le sud depuis deux mille ans. Il faut remarquer que les résultats de ces recherches ont démontré l'existence de deux phénomènes liés à la mousson d'été sud-américaine, tout au long de l'époque géologique signalée : des sous-périodes ont présenté des précipitations vraisemblablement faibles dans la région entre 10000 et 9200,7000 et 5000 , et 1500 et 900 ans avant le présent, d'autres des précipitations vraisemblablement fortes autour de 5000 , entre 2200 et 1500, et 550 et 130 ans avant le présent (Bird et al. 2011b). Même s'il est risqué d'appliquer automatiquement ces données à la côte nord-péruvienne pour avoir une idée des évolutions paléo-climatiques régionales, il est en tout cas possible de remarquer qu'à partir des années 500 apr. J.-C., il y a eu un changement dans les niveaux pluviométriques dans 
l'aire andine. Une période de sécheresse a vraisemblablement affecté alors le monde mochica. Les travaux de Bird et al. (2011a) ont en tout cas démontré que, lors de la période comprise entre 0 et 800 apr. J.-C., l'ITCZ a occupé des positions nettement septentrionales par rapport au millénaire dernier (McCormac et al. 2002, 2004). Ces résultats et les arguments développés par León (2007), Grobman et al. (2012), Lockard (2009) et Ogburn (2012), favorisent l'emploi de la courbe ShCa104. Cependant, Hogg et al. (2013) ont proposé une courbe nouvelle visant à améliorer les calibrations des dates ${ }^{14} \mathrm{C}$ provenant de l'hémisphère sud, la courbe ShCal13. Cette dernière est, à notre avis, la plus pertinente à utiliser dans les études comparatives (voir Reimer et al. 2013 ; Koons et Alex 2014). Il faut toutefois tenir compte d'autres limites méthodologiques, à savoir l'« effet réservoir » et le phénomène des « vieux bois ».

\section{L'effet réservoir}

Les étendues d'eau terrestre sont des réservoirs de carbone échangeable, dont l'essentiel est présent sous la forme de carbone inorganique dissous (Dutta 2008). Par ailleurs, les eaux de surface des océans ont deux sources de ${ }^{14} \mathrm{C}$, l'atmosphère et les eaux profondes. Ces dernières apportent des quantités variables de ${ }^{14} \mathrm{C}$ aux eaux superficielles lorsqu'elles remontent à la surface, selon le phénomène dénommé " upwelling ». Ce phénomène n'est pas uniforme à l'échelle globale, car il dépend de divers facteurs comme la latitude, la topographie des océans, la forme de la ligne côtière, etc. Dans ce cadre, les êtres vivants ayant grandi dans un milieu où ils ont absorbé $d u{ }^{14} \mathrm{C}$ provenant de l'atmosphère et d'un autre réservoir de carbone seront chronologiquement différents (de manière apparente) au moment où on les datera et où on voudra les comparer $a$ posteriori (Stuiver et al. 1986, p. 980 ; Mangerud et al. 2006, p. 3228).

Dans le cas de datations d'êtres marins par le radiocarbone, il faut donc mettre en place une correction en recalibrant la datation avec une autre courbe (par exemple la courbe Marine13), car les organismes marins ont des quantités appauvries en ${ }^{14} \mathrm{C}$ par rapport aux organismes terrestres. Ce phénomène est connu sous le nom « d'effet réservoir 》 et il a été mis en évidence par Jan Mangerud $\left(1972\right.$, p. 151, 154, 156) dans ses recherches sur l'évaluation des dates ${ }^{14} \mathrm{C}$ des coquillages marins modernes en Norvège. Pour le Pérou, les recherches d'Ortlieb et al. (2011) et Kennett et al. (2002) sont à retenir spécialement pour leur contribution à la compréhension de cet effet sur les dates d'échantillons marins et archéologiques de la période archaïque provenant de la côte sud-péruvienne.

Le « vieillissement» apparent est lié aux variations du réservoir marin et de l'âge réservoir. Il provient de l'appauvrissement de l'activité de l'isotope ${ }^{14} \mathrm{C}$ dans les eaux océaniques profondes (c'est-à-dire à un ralentissement de sa désintégration radioactive) ainsi qu'au mélange, incomplet, des eaux profondes avec les eaux superficielles des océans (Mangerud 1972 ; Ulm 2006). 
L'effet réservoir marin régional est noté $\Delta \mathrm{R}$ et l'expression mathématique pour définir $\Delta \mathrm{R}$ est $\Delta \mathrm{R}=\mathrm{P}-\mathrm{Q}(\mathrm{Ulm} 2006, \mathrm{p}$. 57) ou bien $\Delta \mathrm{R}(\mathrm{s})=\mathrm{Rs}(\mathrm{t})-\mathrm{Rg}(\mathrm{t})$ (Stuiver et al. 1986, p. 982). Dans ces formules, $\Delta \mathrm{R}$ est la différence entre une date ${ }^{14} \mathrm{C}$ conventionnelle (P ou Rs $[\mathrm{t}]$ ) d'un échantillon, dont on connaît bien l'âge et l'origine, et une date équivalente ( $\mathrm{Q}$ ou $\mathrm{Rg}[\mathrm{t}])$ produite à partir de l'utilisation d'une courbe, dite marine, paramétrée et modelée à une échelle globale. Selon Kennett et al. (2002, p. 54), le $\Delta$ R pour le Pérou est $190 \pm 40$ années.

L'âge réservoir est la différence entre l'âge des organismes marins et l'âge des organismes terrestres contemporains (Olsen et al. 2010, p. 635). Cet âge n'est pas constant aux échelles temporelle et géographique (Mangerud et Gulliksen 1974 ; Ortlieb et al. 2011, p. 91). Dans les eaux superficielles, il peut varier de 400 ans selon Olsen (Olsen et al. 2010, p. 635) ou entre 350 et 1500 ans selon Mangerud et al. (2006, p. 3228). Dans les eaux profondes, cet âge évolue par rapport à l'échange de $\mathrm{CO} 2$ atmosphérique, l'afflux des eaux douces alourdies et l' " upwelling » (Olsen et al. 2010, p. 635).

En raison de l'effet réservoir, les datations des populations qui ont eu des régimes alimentaires à base de produits marins, apparaissent plus anciennes que celles dont les régimes alimentaires comprenaient exclusivement des produits terrestres (voir Olsen et al. 2010, p. 635).

Il faut encore noter ici le manque d'études sur l'effet réservoir sur la côte nord du Pérou et le manque de recherches systématiques comparatives sur les régimes alimentaires des populations à l'échelle régionale et/ou étatique mochica (voir, à titre d'exemple, Russell et Jackson 2001, p. 164-165 [pour le site de Cerro Mayal dans la vallée de Chicama]). Cette situation rend toute étude des datations de notre zone difficile car la consommation de produits marins par les sociétés côtières peut avoir modifié les dates que l'on obtient sur des vestiges humains. Dans l'inventaire des échantillons analysés ici, il n'en existe pas d'origine marine. Un seul est peut-être d'origine mixte, c'est-à-dire à la fois marine et terrestre (Beta-108281). On ne l'a pas exclu, mais il a été recalibré en utilisant la courbe Marine13.

\section{Les vieux bois}

En 2014, la recherche archéologique menée sur le site de San José de Moro, dans la vallée de Jequetepeque, a permis aux archéologues de vérifier l'usage d'une essence locale appelée algarrobo (Prosopis sp.) pour la construction des toits des chambres funéraires des élites, pendant la période Mochica tardive. Cette essence fait encore partie du paysage désertique côtier péruvien et elle est actuellement utilisée comme carburant dans les fours servant à cuire des céramiques locales dans la province de Chepén. Par ailleurs, sur le site de Huacas de Moche, Moutarde (2008) a mis en évidence plusieurs essences employées dans la vie quotidienne et rituelle mochica. C'est le cas de la canne de Guayaquil 
(Guadua angustifolia), du lúcumo (Pouteria lucuma), de l'algarrobo qui ont été couramment utilisés comme poutres ; la caña brava (Gynerium sagittatum), pour sa part, a été utilisée pour la construction de cercueils, de murs et de toits (Moutarde 2008, p. 304).

Matériaux vivants ou, plus exactement, issus du vivant, le bois et le charbon de bois sont des vestiges souvent abondants dans les sites mochica. Ils ont été couramment sélectionnés, échantillonnés et datés par le radiocarbone dans le cadre de recherches à objectif chronologique. Cependant la datation du bois - ou de charbon de bois - butte sur des limites méthodologiques : elle n'est pas liée au moment de l'abattage, mais à l'anneau de croissance de l'essence qui forme l'échantillon. Pour accepter une date ${ }^{14} \mathrm{C}$ provenant d'un arbre, il faut contrôler plusieurs facteurs : la durée de vie de l'espèce, sa longévité (ainsi, Skolmen [1990] a étudié un algarrobo de plus de 100 ans à Hawai), la partie de l'essence datée (de fait, la partie externe d'un bois sera très probablement moins ancienne que la partie interne) et la réutilisation ou le réemploi de matériaux anciens. Les distorsions dues à la datation de bois ou de charbons de bois sont connues sous l'appellation « vieux bois » (voir Kennett et al. 2002 [pour la côte sud-péruvienne] ; Scharf et al. 2013 [pour l'Asie] ; Caracuta et al. 2012 [pour l'Italie], etc.).

Dans notre corpus, $17 \%$ des dates inventoriées correspondent à des échantillons de bois et $32 \%$ à des échantillons de charbons (voir infra). Environ $50 \%$ des échantillons fournissent donc des informations assez incertaines, car les essences datées n'ont pas été identifiées par les auteurs en raison du manque d'études anthracologiques systématiques (Figure 2). Cela constitue un vrai

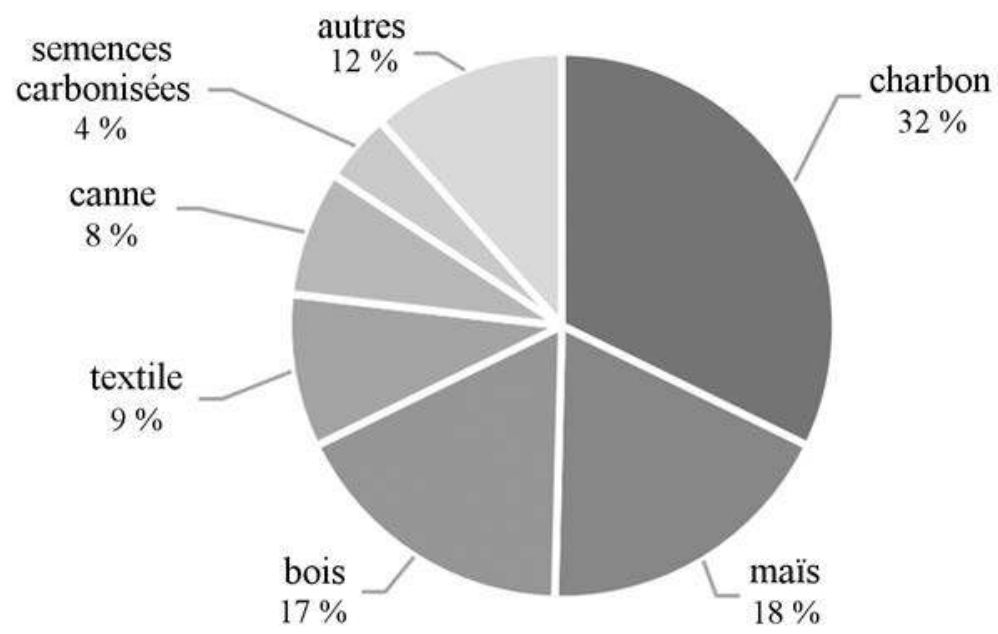

Fig. 2 - Pourcentages relatifs des principaux types de matériaux datés par ${ }^{14} \mathrm{C}$ au sein des contextes mochica. 
problème qui interdit d'examiner la validité des dates obtenues de cette façon par traitement statistique. Cela dit, notre objectif principal est ici de présenter un inventaire relativement complet et mis à jour des dates ${ }^{14} \mathrm{C}$ dont on dispose (en spécifiant la région, le site et les matériaux datés). On s'est gardé en revanche de donner des découpages précis aux phases définies par les typologies céramiques sur la base des dates ${ }^{14} \mathrm{C}$, car ces dernières peuvent être imprécises.

Pour la recalibration des dates, deux approches étaient envisageables. Soit seules les dates obtenues sur d'autres matériaux que le bois ou le charbon de bois étaient traitées et analysées ; soit une analyse exploratoire et globale de tout le corpus était mise en œuvre. C'est la deuxième option que nous avons retenue, car les échantillons des types bois et charbon ne constituent que la moitié du corpus, et c'était en réalité une occasion de tester leur validité. Notre recherche se veut être, par ailleurs, une contribution à la discussion sur la chronologie mochica : on fait ici un état des lieux sans dissimuler les deux limites méthodologiques rappelées ci-dessus. Nous pensons en tout cas que notre démarche pourra améliorer à l'avenir le choix des protocoles de sélection des échantillons à dater. Même s'il n'existe pas à ce jour de travaux sur les vieux bois et l'effet réservoir et leurs conséquences dans la chronologie mochica, il apparaît indispensable d'effectuer des échantillonnages nouveaux sur des matériaux carbonisés de courte vie (Rick et al. 2005, p. 1641) pour obtenir des dates qui minimiseront les risques d'erreurs mentionnés.

\section{Résultats}

Dans le cas de la sous-aire Mochica de Piura, un seul échantillon - provenant du site de Loma Negra - daté par ${ }^{14} \mathrm{C}$ (Lechtman et al. 1982, p. 5 [I-5573]) figure à notre inventaire. Il s'agit d'un fragment de fût de bois. L'échantillon a été recueilli en 1971 et il a produit une date de $1655 \pm 95$ ans (240-635 apr. J.-C. [2 $\sigma]$ ). Lechtman et al. (1982) ont hypothétiquement relié cette date au commencement de la phase Mochica II.

Sur un autre plan il faut observer qu'aucun échantillon d'origine marine n'est apparu dans la littérature révisée ; en revanche, deux échantillons d'origine mixte ont été répertoriés : Beta-108281 et Beta-1568907 (Uceda et al. 2001, p. 216). Dans la source consultée, Beta-1568907 n'est pas relié à l'une des phases de Larco. L'échantillon Beta-108281, lui, correspondrait chronologiquement à la phase Mochica IV. Cette date doit toutefois être prise avec précaution à cause du possible vieillissement des datations sur ossements et coquillages (effet réservoir : voir supra). On a donc calibré cette date en utilisant deux courbes, ShCal13 et Marine13. L'emploi de la courbe ShCal13 a produit des dates bien anciennes pour un contexte Mochica IV à Huacas de Moche (203-390 apr. J.-C. [2 $\sigma, 94,1 \%])$. En revanche, la courbe Marine13 a produit des résultats plus 
cohérents et qui peuvent être reliés à d'autres datations de la phase Mochica IV (518-695 apr. J.-C. [2 б]).

Certaines données n'ont pas été prises en compte pour le traitement statistique. Par exemple, Dillehay et Kolata (2004) ont publié deux dates ${ }^{14} \mathrm{C}$ pour la vallée de Jequetepeque ${ }^{2}$. Elles ne sont pas incluses ici, car elles ne sont pas reliées aux phases stylistiques de Larco. Pour la même sous-aire, Rosas (2007) présente des dates déjà calibrées associées à la période Mochica tardive du site archéologique Cerro Chepén ${ }^{3}$. Elles n'ont pas été retenues, car les dates avant le présent ne sont pas données.

Toutes les informations que nous avons inventoriées ont été regroupées dans les Figures 3, 4 et 5 pour la sphère Mochica nord et dans les Figures 6, 7, 8, 9 pour la sphère Mochica sud.

2. Données ${ }^{14} \mathrm{C}$ dans Dillehay et Kolata (2004, p. 4327) : échantillon : Beta-143883, site : JE-125, âge ${ }^{14} \mathrm{C}: 1520 \pm 60$ ans ; échantillon : Beta-143885, site : JE-339, âge ${ }^{14} \mathrm{C}: 1370$ \pm 70 ans.

3. Données ${ }^{14} \mathrm{C}$ dans Rosas $(2007$, p. 235$)$ : échantillon : AA61865, date calibrée : 790 \pm 100 ans apr. J.-C. ; échantillon : AA61870, date calibrée : $780 \pm 100$ ans apr. J.-C. ; échantillon : AA61866, date calibrée : $780 \pm 115$ ans apr. J.-C. 

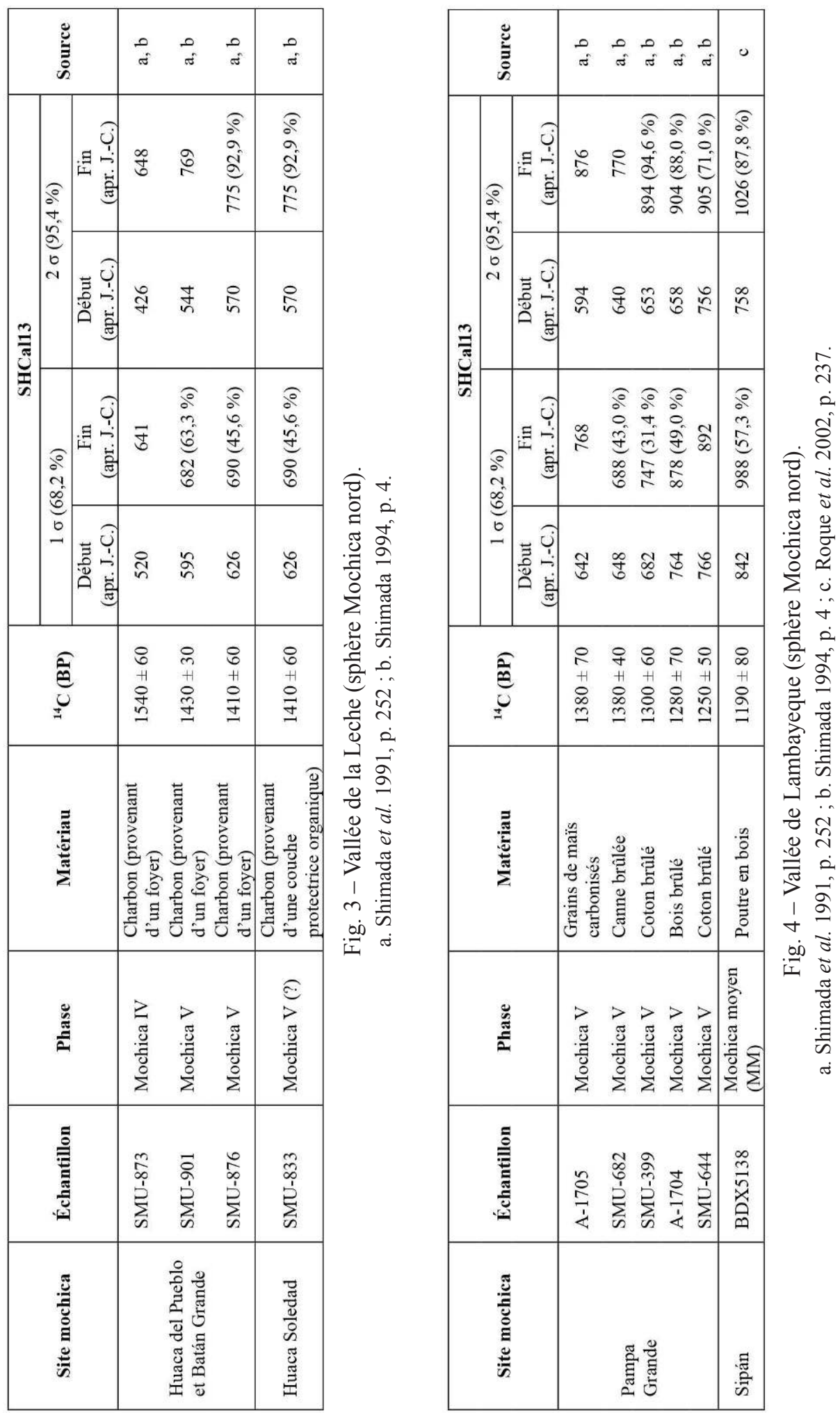


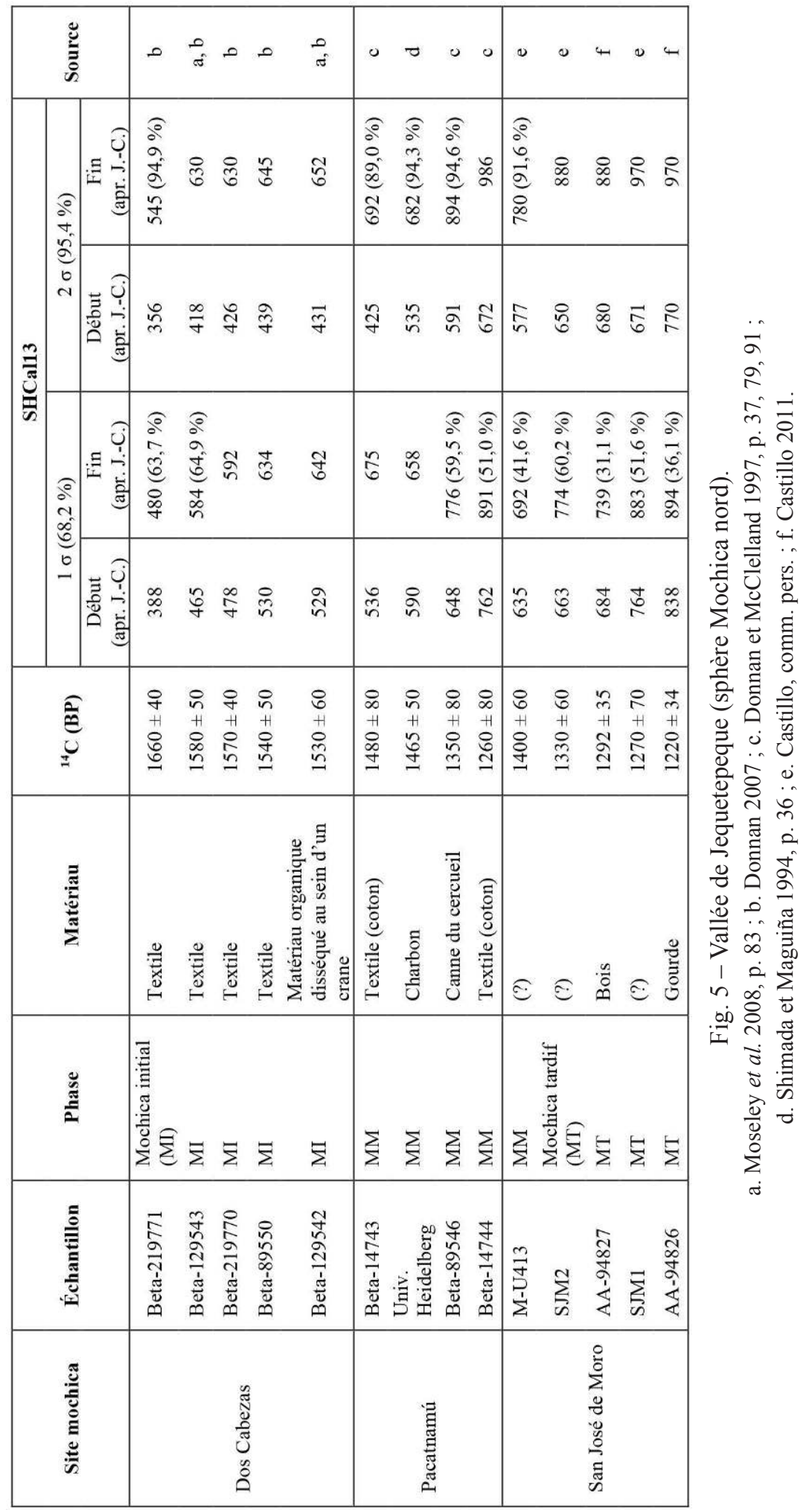




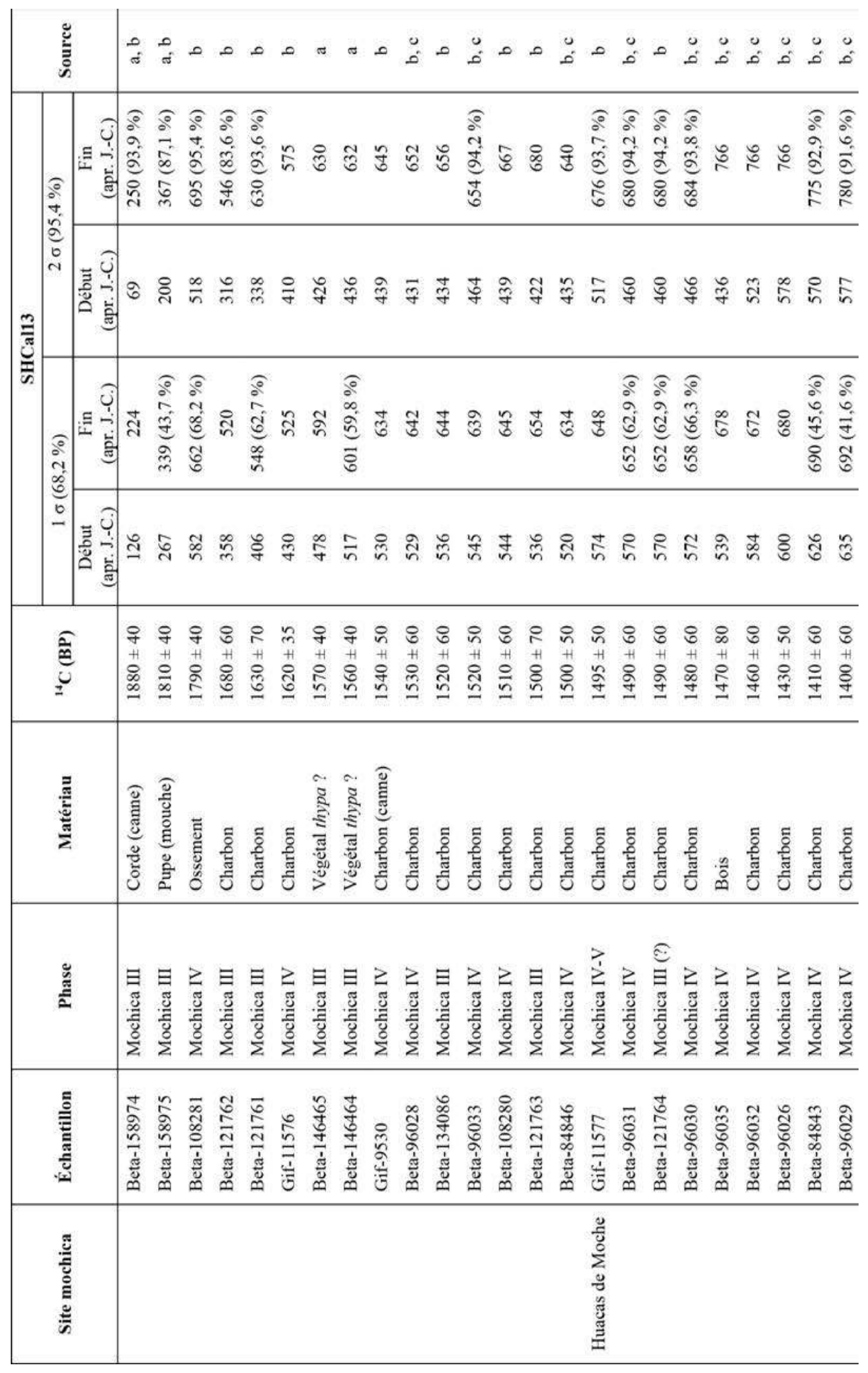




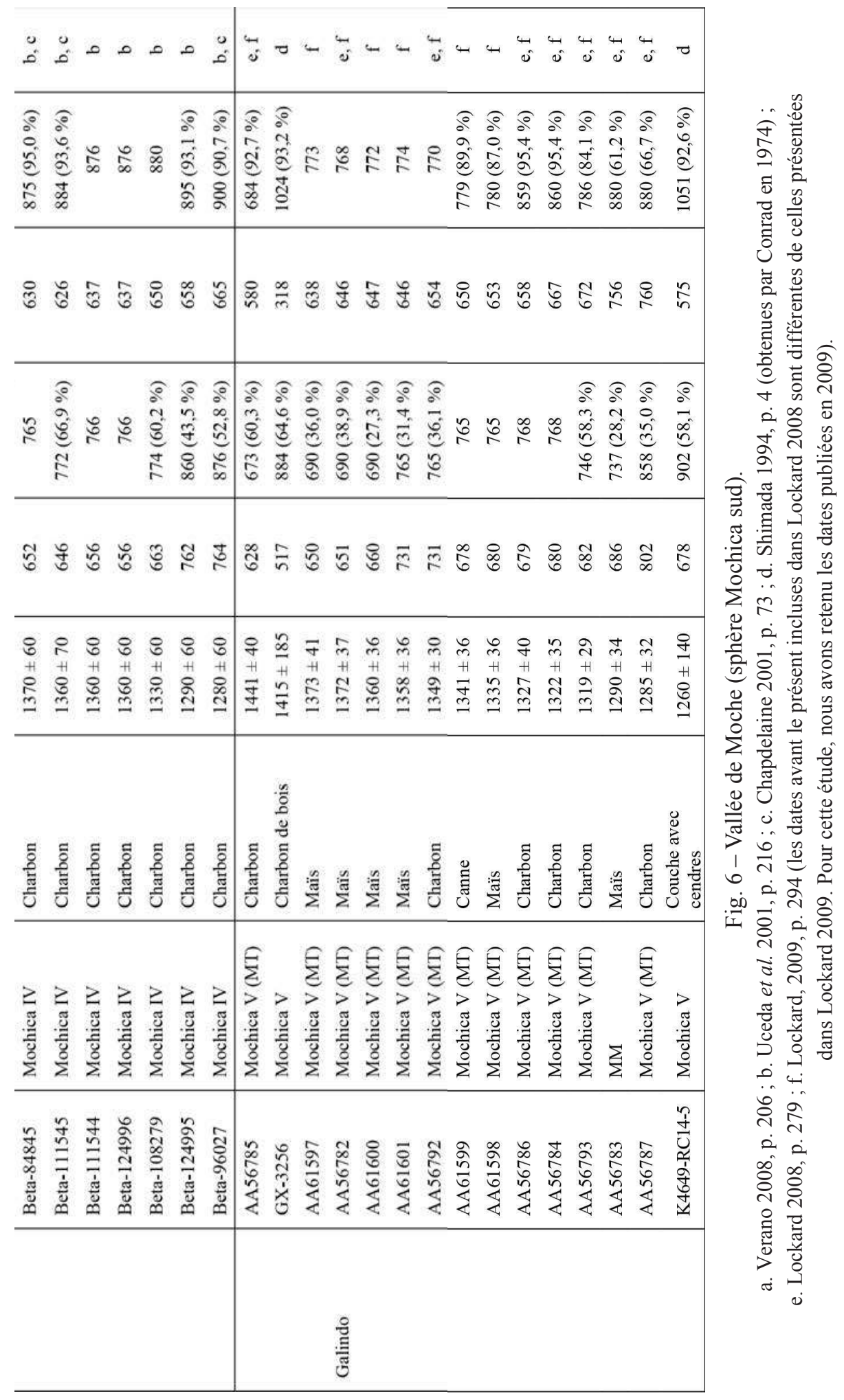




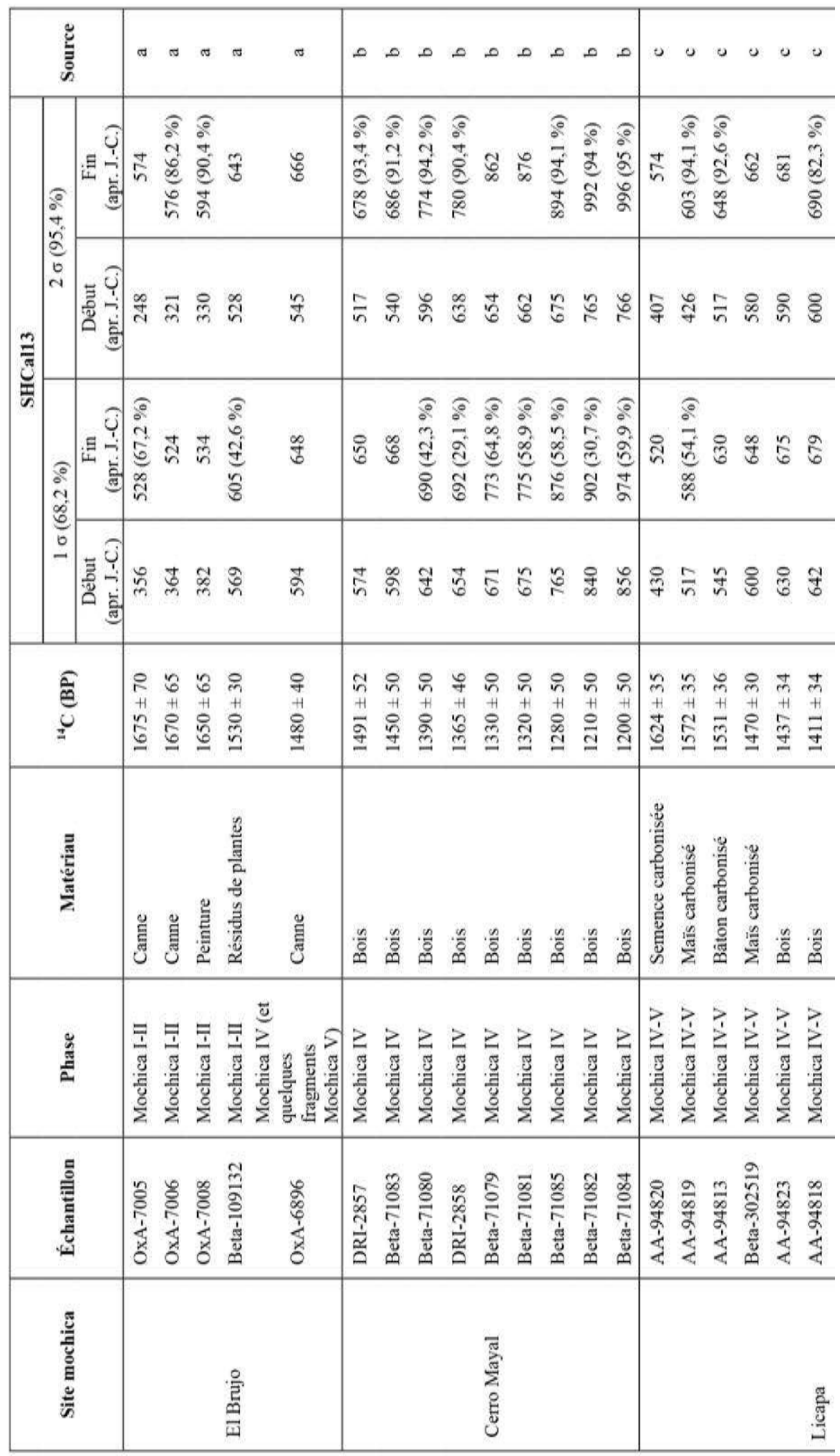



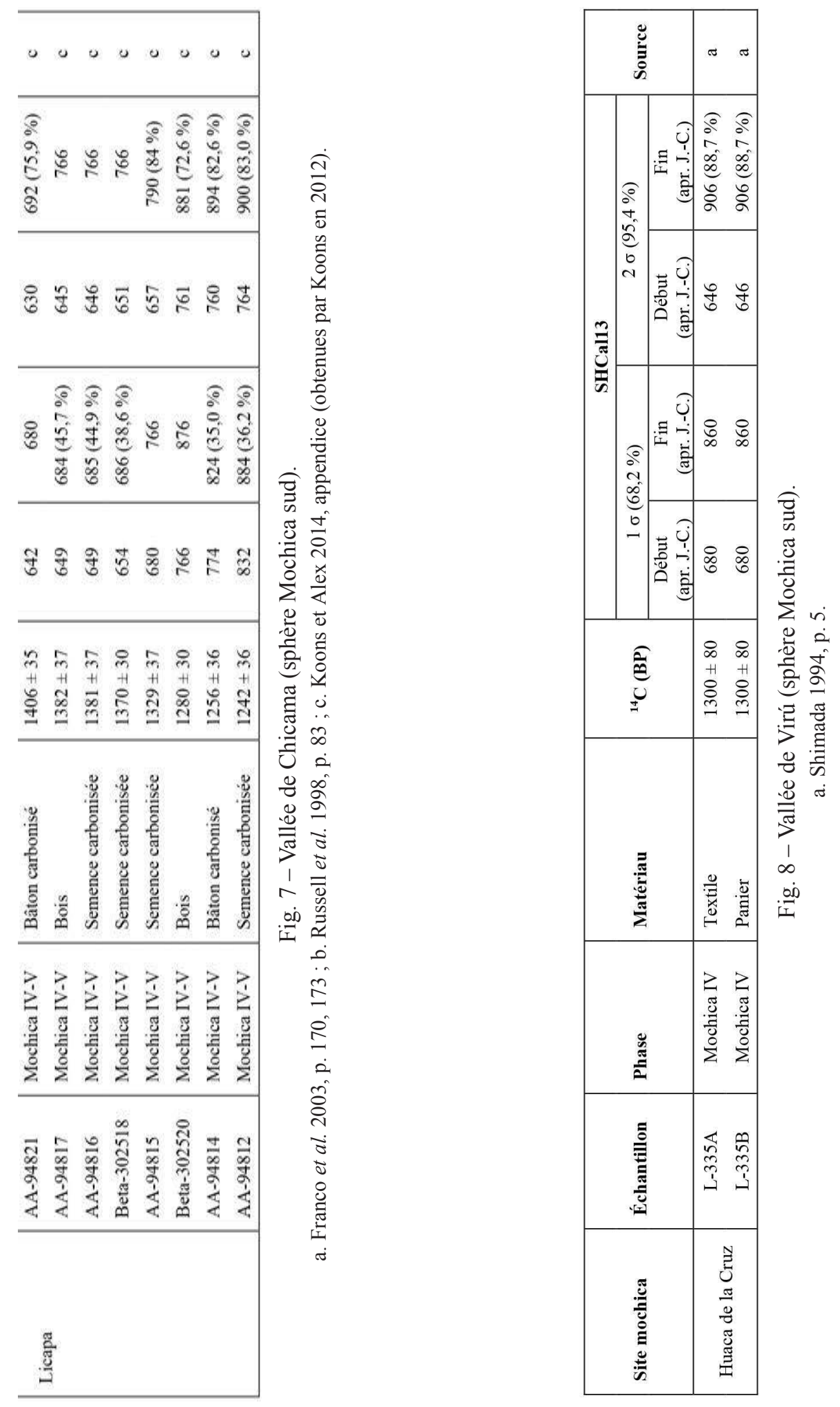


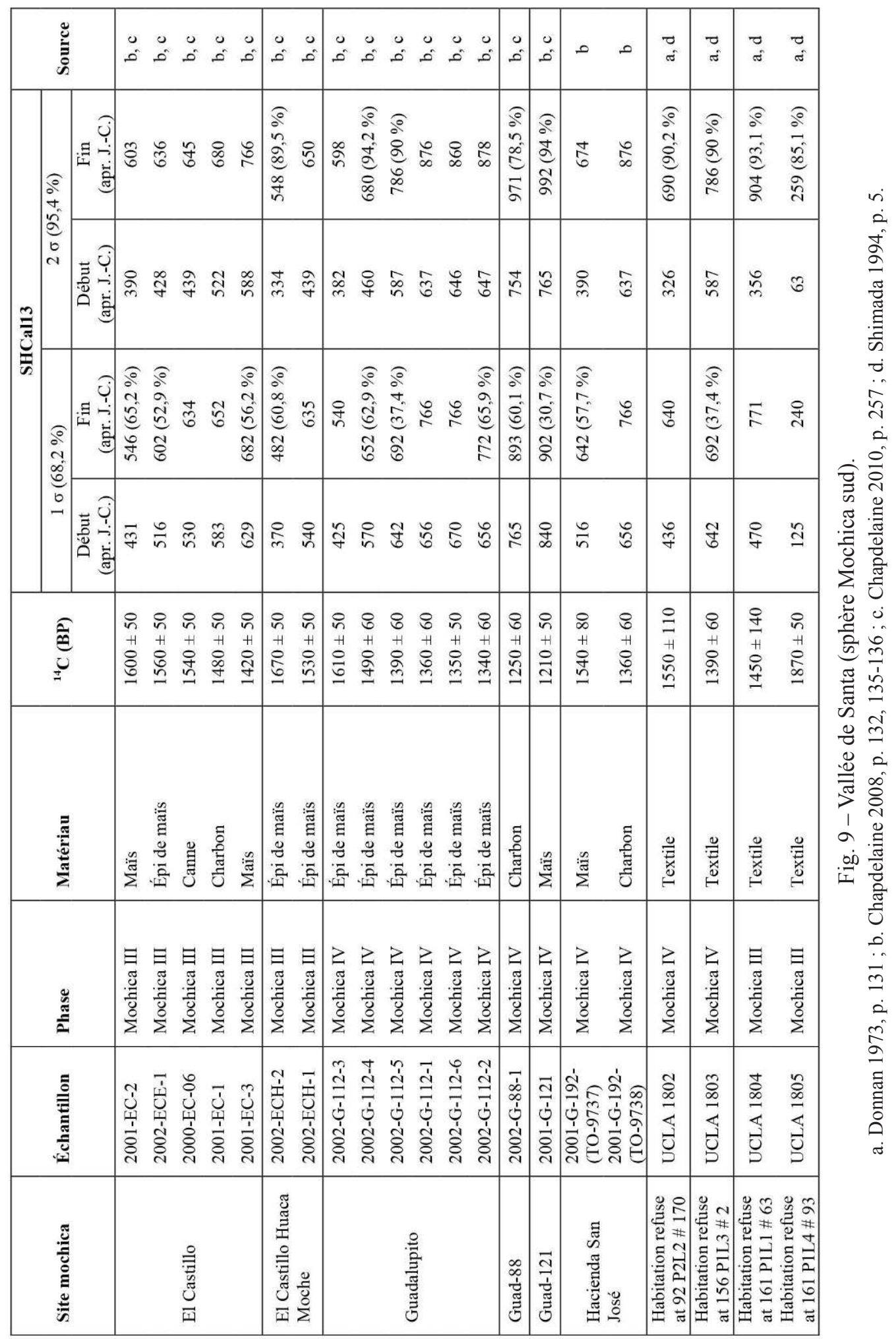




\section{Discussion}

L'un des enjeux de l'archéologie mochica est la comparaison des données (typologiques et chronologiques) à l'échelle des deux grandes sphères distinguées : «La séquence céramique est l'approche fondamentale pour mettre en place la chronologie mochica ; en même temps, les dates radiocarbone doivent être comprises comme des outils servant à améliorer la connaissance du cadre culturel mochica. La sériation de la céramique vallée par vallée est l'une des solutions (Castillo 2000, 2003), mais une comparaison inter-vallées et la recherche d'une unité dans la description des céramiques sont aussi nécessaires » (Chapdelaine 2011, p. 195 [traduit de l'anglais]).

Concernant la quantité des datations répertoriées sphère par sphère, on remarquera qu'environ $20 \%$ seulement des dates proviennent de l'aire Mochica nord. Dans cet espace, $65 \%$ des datations ne proviennent pas d'échantillons du type bois ou charbon, ce qui est assez positif pour les prochaines études comparatives. Malgré tout, les datations des sites de la région nord (surtout Dos Cabezas, Pacatnamú et San José de Moro) devront être augmentées afin d'assurer une meilleure compréhension de l'évolution et de la diffusion des styles dans cette région. Dans la sphère sud, la moitié des datations provient d'échantillons de bois et/ou de charbon. L'autre moitié correspond à d'autres matériaux ; presque $30 \%$ des dates ont été obtenus à partir de plantes comme le maïs (Zea mays) et les cannes (Guadua angustifolia et/ou Gynerium sagittatum).

Ces éléments précisés, une première constatation s'impose : même si les risques concernant les vieux bois ne sauraient être sous-estimés, l'utilisation de l'ensemble des datations fait apparaître des différences chronologiques significatives entre les phases III et IV. Il existe ainsi une différence chronologique entre l'apparition de la phase Mochica III (sur les sites de Huacas de Moche, El Castillo, El Castillo Huaca Moche Habitation refuse at 161 P1L1 \# 63 ; et Habitation refuse at 161 P1L4 \# 93) et celle de la phase Mochica IV (dans le site de Huacas de Moche) (voir Figure 10).

\begin{tabular}{|c|c|}
\hline Période (nombre de dates) & Période (nombre de dates) \\
\hline aMS-III (n = 17) & aMS-IV $(n=21)$ \\
\hline \multicolumn{2}{|c|}{$\mathrm{p}$ (same) : 6.874E-05 } \\
\hline
\end{tabular}

Fig. 10 - Comparaison des dates ${ }^{14} \mathrm{C}$ calibrées $(2 \sigma)$ correspondant à l'apparition des périodes Mochica sud-phase III (aMS-III) et Mochica sud-phase IV (aMS-IV) (Test Mann-Whitney).

Afin d'apporter des précisions chronologiques nouvelles, nous avons commencé à développer des comparaisons aux échelles intersite et intervallée. Les premières données ne sont pas encore concluantes, mais elles peuvent servir à 
proposer des hypothèses de travail. Ces résultats nous permettent notamment de constater l'insuffisance de données radiométriques concernant l'origine temporelle du phénomène mochica dans les deux sphères. Dans le cas des échantillons rattachés à un contexte archéologique de la phase Mochica initiale, les dates les plus anciennes se situent autour des années 250-350 apr. J.-C. (sites de Dos Cabezas et El Brujo). Mais les données pour cette période sont rares et cela constitue un véritable problème (Chapdelaine 2011).

Concernant les autres phases, des données hétérogènes selon les vallées et les sphères ont été avancées. Selon Chapdelaine (2011, p. 195), les deux phases III et IV du Sud peuvent coïncider avec la phase Mochica moyenne du Nord. Statistiquement, l'apparition de la phase Mochica III dans la sphère sud (représentée par les datations obtenues à Huacas de Moche, El Castillo, El Castillo Huaca Moche Habitation refuse at 161 P1L1 \# 63 et Habitation refuse at 161 P1L4 \# 93) diffère significativement, d'un point de vue chronologique, de l'apparition de la phase Mochica moyenne de la sphère nord (représentée par les datations obtenues à Pacatnamú, Sipán et San José de Moro) (voir Figure 11).

\begin{tabular}{|c|c|}
\hline Période (nombre de dates) & Période (nombre de dates) \\
\hline $\mathrm{aMS}-\mathrm{III}(\mathrm{n}=17)$ & $\mathrm{aMM}(\mathrm{n}=6)$ \\
\hline \multicolumn{2}{|r|}{$\mathrm{p}($ same $): 0.004564$} \\
\hline
\end{tabular}

Fig. 11 - Comparaison des dates ${ }^{14} \mathrm{C}$ calibrées $(2 \sigma)$ correspondant à l'apparition des périodes Mochica sud-phase III (aMS-III) et Mochica moyenne (aMM) (Test Mann-Whitney).

Au contraire, le moment d'apparition de la phase IV (dans les sites de Huacas de Moche, Cerro Mayal, Huaca la Cruz, Guadalupito, Guad-88, Guad-121 et Hacienda San José) est significativement similaire à celui de la phase Mochica moyenne (dans les sites de Pacatnamú, Sipán et San José de Moro) (voir Figure 12).

\begin{tabular}{|c|c|}
\hline Période (nombre de dates) & Période (nombre de dates) \\
\hline $\mathrm{aMS}-\mathrm{IV}(\mathrm{n}=44)$ & $\mathrm{aMM}(\mathrm{n}=6)$ \\
\hline \multicolumn{2}{|c|}{$\mathrm{p}($ same $): 0.8112$} \\
\hline
\end{tabular}

Fig. 12 - Comparaison des dates ${ }^{14} \mathrm{C}$ calibrées $(2 \sigma)$ correspondant à l'apparition des périodes Mochica sud-phase IV (aMS-IV) et Mochica moyenne (aMM) (Test Mann-Whitney).

Pour ce qui est de la contemporanéité du phénomène de transition stylistique correspondant aux phases III et IV dans les sites de la région Mochica sud, la question se pose en particulier de savoir s'il existe une différence significative 
ou non entre les datations relatives à la fin de la phase III dans la vallée de Moche et dans celle de Santa. Existe-t-il, par ailleurs, une cohérence ou une différence significative entre les datations relatives à l'apparition de la phase IV dans la vallée de Moche et dans celle de Santa ? En appliquant toujours le test de Mann-Whitney, il est possible de confirmer que les dates moyennes pour la fin de la phase III et l'apparition de la phase IV dans ces deux vallées sont similaires (voir Figure 13).

\begin{tabular}{|c|c|}
\hline Période (nombre de dates) & Période (nombre de dates) \\
\hline $\mathrm{dMS}-\mathrm{III}-\mathrm{M}(\mathrm{n}=8)$ & $\mathrm{aMS}-\mathrm{IV}-\mathrm{S}(\mathrm{n}=12)$ \\
\hline \multicolumn{2}{|c|}{$\mathrm{p}($ same $): 0.7282$} \\
\hline
\end{tabular}

Fig. 13 - Comparaison des dates ${ }^{14} \mathrm{C}$ calibrées $(2 \sigma)$ correspondant à la disparition de la période Mochica sud-phase III-vallée de Moche (dMS-III-M) et à l'apparition du style Mochica sud-phase IV-vallée de Santa (aMS-IV-S) (Test Mann-Whitney).

Statistiquement, il est donc possible de corroborer l'existence d'un haut degré de cohésion chrono-stylistique - aux niveaux de la décoration et des formes céramiques - entre ces vallées, même pendant cette période de transition. Enfin, l'analyse comparative menée sur les échantillons de la sphère sud et de la phase IV, démontre que l'apparition et la fin de ce style dans les sites de Huacas de Moche, Cerro Mayal, Guadalupito, Guad-88, Guad-121 et Hacienda San José sont des phénomènes probablement contemporains (voir Figures 14, $15,16 \mathrm{a}$ et $16 \mathrm{~b}$ [page suivante]).

\begin{tabular}{|c|c|}
\hline Période (nombre de dates) & Période (nombre de dates) \\
\hline aMS-IV-HM (n = 21) & aMS-IV-CM (n = 9) \\
\hline \multicolumn{2}{|c|}{$\mathrm{p}$ (same) : 0.007569} \\
\hline
\end{tabular}

Fig. 14 - Comparaison des dates ${ }^{14} \mathrm{C}$ calibrées $(2 \sigma)$ correspondant à l'apparition de la phase IV dans le site de Huacas de Moche (aMS-IV-HM) et dans celui de Cerro Mayal (aMS-IV-CM) (Test Mann-Whitney).

\begin{tabular}{|c|c|}
\hline Période (nombre de dates) & Période (nombre de dates) \\
\hline aMS-IV-GHSJ $(\mathrm{n}=12)$ & aMS-IV-CM $(\mathrm{n}=9)$ \\
\hline $\mathrm{p}($ same $)$ & $: 0.1352$ \\
\hline
\end{tabular}

Fig. 15 - Comparaison des dates $2 \sigma$ correspondant à l'apparition de la phase IV dans les sites de Guadalupito, Guad-88, Guad-121 et Hacienda San José (aMSV-GHSJ) et dans celui de Cerro Mayal (aMS-IV-CM) (Test Mann-Whitney). 

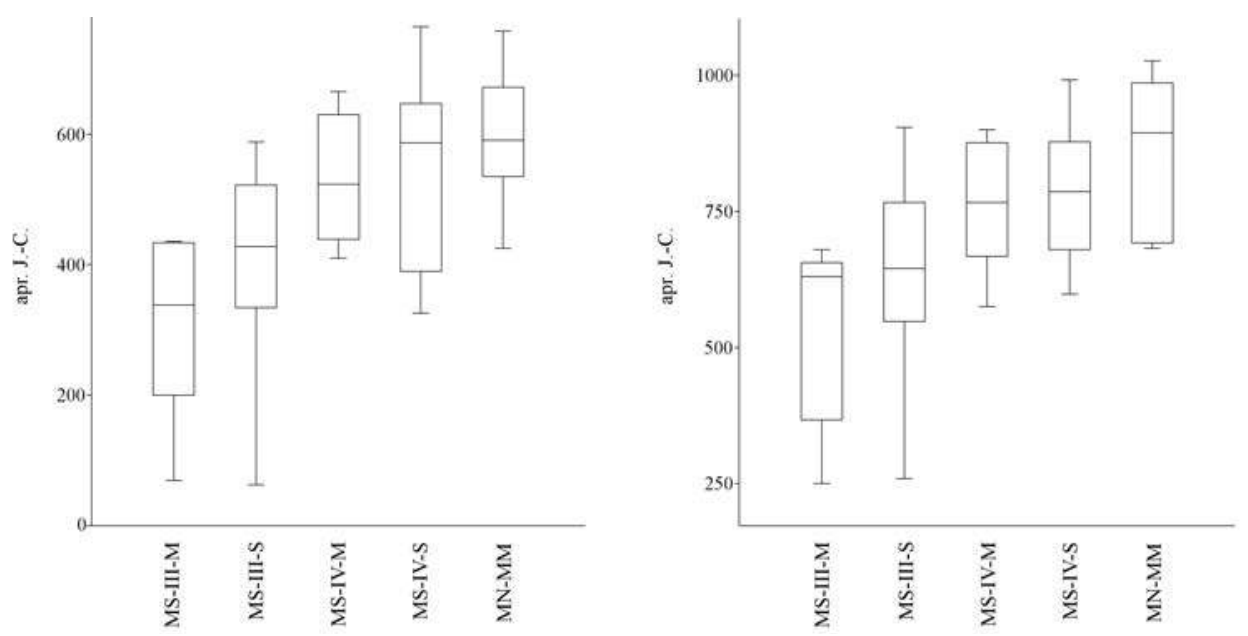

Fig. $16 \mathrm{a}$ - Boîtes à moustaches montrant le regroupement des dates ${ }^{14} \mathrm{C}$ calibrées $(2 \sigma)$ relatives à l'apparition des phases céramiques III, IV et Mochica moyen. La ligne à l'intérieur d'une boîte indique la médiane des données.Chaque boîte est délimitée par les $1^{\circ}$ et $3^{\circ}$ quartiles.Fig. $16 \mathrm{~b}$ - Boîtes à moustaches montrant le regroupement des dates ${ }^{14} \mathrm{C}$ calibrées $(2 \sigma)$ relatives à la disparition des phases III, IVet Mochica moyen. La ligne à l'intérieur d'une boîte indique la médiane des données. Chaque boîte est délimitée par les $1^{\circ}$ et $3^{\circ}$ quartiles.

$\begin{aligned} \text { Légende : } & \text { IV phase IV } \\ \text { MS Mochica sud } & \text { MM Mochica moyenne } \\ \text { MN Mochica nord } & \text { S vallée de Santa } \\ \text { III phase III } & \text { M vallée de Moche }\end{aligned}$

À propos des dernières occupations mochica, il apparaît qu'il est encore nécessaire d'effectuer davantage de prospections archéologiques afin de répertorier des sites nouveaux qui présenteraient des occupations Mochica V dans la sphère sud. De la même manière, il serait pertinent d'accroître le nombre de datations issues de contextes Mochica tardif dans la sphère nord.

Dans le site de Pampa Grande (section nord), les vestiges céramiques ont toujours été caractérisés comme Mochica $V$ en raison de leurs attributs stylistiques (forme et décoration) : «Pampa Grande a été le site archéologique mochica le plus grand, le plus diversifié et le plus complexe. La plupart des échantillons céramiques trouvés en surface correspondent à une occupation Mochica $\mathrm{V}$, la dernière des Moche... » (Anders 1981 [traduit de l'anglais]). Le site de San José de Moro (également au nord) présente des occupations, des styles céramiques nettement tardifs et de l'influence des sociétés wari et cajamarca. Cette influence se marque d'abord par des décors qui ont été importés sur le site. Mais c'est aussi 
vrai pour de nouvelles formes (comme les vases à deux goulots ou de nouvelles bouteilles et vases peints portant des décorations polychromes) qui font partie du registre archéologique des périodes Mochica tardif et transitionnel (Castillo 2000). En réalité, des influences de type technologique entre ces trois sociétés commencent aussi à être évoquées (Nickel et al. 2013). On a pu, par exemple, remarquer l'usage d'oxydes de manganèse pour la décoration peinte des vases wari (Del Solar 2011). Il s'agit d'une technique employée également à San José de Moro pour décorer des bouteilles hybrides mochica-wari (Dollwetzel 2012) vraisemblablement à partir de la phase Mochica tardif $\mathrm{B}$.

Entre Pampa Grande et San José de Moro, il existe une corrélation chronologique et stylistique, même si l'apparition de la phase Mochica tardif à Pampa Grande semble être visuellement plus ancienne.

En fait, le décalage entre les données de ces deux sites et les données obtenues à la Huaca del Pueblo, ainsi que la comparaison des informations disponibles à une échelle macro-régionale, soulignent la nécessité de continuer la construction d'un corpus général de données radiométriques mochica. Encore une fois, en appliquant le test Mann-Whitney, les dates correspondant à la fin des styles Mochica tardif (à Pampa Grande) et Mochica V (à Galindo) ne sont pas différentes. Dans ce contexte, il est possible d'affirmer que la valeur de la variable chronologique ne change pas en fonction du site étudié, même quand la fin du style Mochica V semble être légèrement plus récente à Galindo que sur les sites de la sphère nord: Figures 17, 18a et 18b (page suivante).

\begin{tabular}{|c|c|}
\hline Période (nombre de dates) & Période (nombre de dates) \\
\hline dMS-V-GA $(\mathrm{n}=13)$ & dMN-V-PG $(n=5)$ \\
\hline \multicolumn{2}{|c|}{$\mathrm{p}$ (same) : 0.1037} \\
\hline
\end{tabular}

Fig. 17 - Comparaison des ${ }^{14} \mathrm{C}$ calibrées $(2 \sigma)$ correspondant à la fin des styles Mochica V à Galindo (dMS-V-GA) et Mochica tardif

(V) à Pampa Grande (dMN-V-PG) (Test Mann-Whitney).

En définitive, la fin des styles Mochica $\mathrm{V}$ et Mochica tardif est parallèle au phénomène de fin lente et progressive de ces sociétés sur la côte nord du Pérou, entre le $\mathrm{IX}^{\mathrm{e}}$ et le $\mathrm{X}^{\mathrm{e}}$ siècle de notre ère. À une échelle macro-régionale, la cause de l'effondrement sociétal a été probablement triple (Castillo 2003) : l'instabilité de l'environnement, l'influence de sociétés étrangères (par exemple wari et cajamarca) et un écroulement politique d'origine interne. Ainsi, les recherches menées à Pampa Grande (Shimada 1994), à Galindo (Bawden 1982) et à San José de Moro (Castillo 2003) supportent l'hypothèse d'un stress interne entre classes sociales (sous la forme de révoltes violentes mettant en cause le pouvoir des élites locales et les méthodes d'intégrations sociale et culturelle). Par ailleurs, les informations archéologiques récentes concernant le site de Cerro 

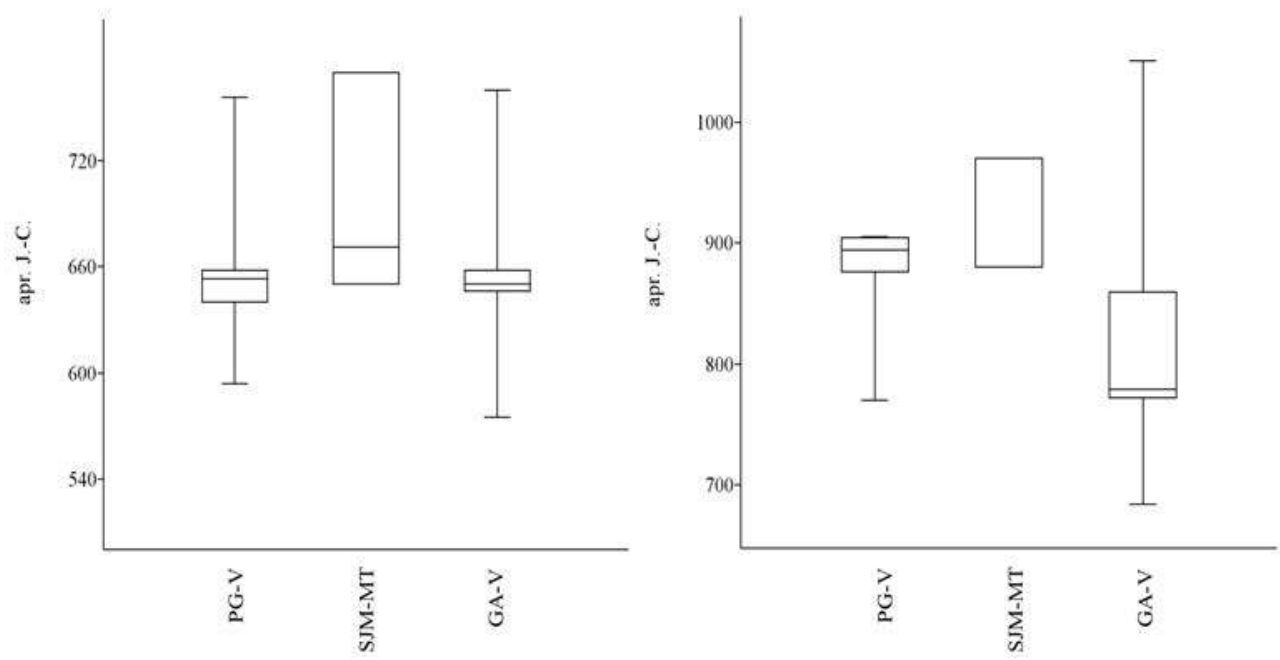

Fig. $18 \mathrm{a}-$ Boîtes à moustaches montrant le regroupement des dates ${ }^{14} \mathrm{C}$ calibrées $(2 \sigma)$ relatives à l'apparition de la phase Mochica V et Mochica tardif dans trois sites archéologiques mochica. La ligne à l'intérieur d'une boîte indique la médiane des données.Fig. $18 \mathrm{~b}$ - Boîtes à moustaches montrant le regroupement des dates ${ }^{14} \mathrm{C}$ calibrées $(2 \sigma)$ relatives à la disparition de la phase

Mochica V et Mochica tardif dans trois sites archéologiques mochica.

$$
\begin{aligned}
\text { Légende : } & \text { PG Pampa Grande (Mochica nord) } \\
\text { GA Galindo (Mochica sud) } & \text { SJ San José de Moro (Mochica nord) }
\end{aligned}
$$

Chepén indiquent que l'effondrement politique des communautés mochica a vraisemblablement aussi résulté de la pression exercée par des groupes étrangers cajamarca (Rosas 2007). En revanche, les données archéologiques provenant d'autres sites comme El Castillo ne montrent pas de processus de violence en rapport avec l'occupation post-mochica (Bélisle 2008).

Un effondrement sociopolitique lié à une instabilité de l'environnement paraît par ailleurs un scénario vraisemblable (Shimada et al. 1991). Les données paléoclimatiques pour la région andine révèlent que des conditions d'aridité ont prévalu entre 500-1100 apr. J.-C. avec un pic entre 900-1100 apr. J.-C. (Bird et al. 2011b, p. 199). Il semblerait que l'apparition de ces longues périodes de sécheresse ait affecté la production agricole des communautés andines et côtières de l'époque. Cela aurait probablement joué un rôle dans la détérioration des relations sociales entre élites locales et non-élites.

Dans tous les cas, des événements d'ordre climatique sont toujours considérés comme ayant eu un rôle déterminant dans les mobilités géographiques des communautés entre les périodes Mochica ancienne et moyenne, et Mochica moyenne et tardive (Castillo 2003). 


\section{Conclusions}

Les sociétés mochica ont occupé et modifié le paysage d'une dizaine de vallées fertiles, séparées par des zones côtières arides, dans la partie septentrionale du Pérou. Aux yeux des andinistes contemporains, les organisations technoculturelle et socio-politique de cette culture sont particulièrement complexes.

L'analyse croisée de données chrono-céramiques et de datations ${ }^{14} \mathrm{C}$ obtenues dans des contextes mochica permet de placer l'apparition de cette société et ses premiers développements entre le $\mathrm{II}^{\mathrm{e}}$ et le $\mathrm{III}^{\mathrm{e}}$ siècle de notre ère. Ayant prospéré probablement autour de systèmes de réciprocité sociale, de cérémonialisme théâtralisé et de valeurs culturelles et ethniques partagées, les sociétés mochica ont disparu entre le $\mathrm{IX}^{\mathrm{e}}$ et le $\mathrm{X}^{\mathrm{e}}$ siècle à cause d'une combinaison de problèmes endogènes et exogènes.

Pour ce qui est des données radiométriques, il est évident que, pour les phases céramiques Mochica I et II ainsi que Mochica initiale, le corpus actuel demeure trop faible pour établir des jalons fermes. L'analyse statistique des dates au radiocarbone qui caractérisent l'interface chronologique des phases III et IV confirme, pour sa part, l'unité chrono-culturelle de l'aire mochica du Sud. Par ailleurs, la quantité et la variabilité des données dans cette zone permettent de penser que l'origine de plusieurs traits culturels et matériels mochica (surtout Mochica III) proviennent de ce secteur et, plus spécifiquement, des Huacas de Moche. En revanche, il n'est pas encore possible de déterminer sur quel site le style Mochica IV est apparu d'abord dans l'aire Mochica sud. Pour la période tardive, l'analyse comparative des datations obtenues à Pampa Grande (secteur Mochica nord) et à Galindo (secteur Mochica sud) indique, au moins présentement, que la fin des styles les plus récents a été un phénomène contemporain.

À l'avenir il conviendra de privilégier la datation de matériaux terrestres de longévité courte. L'obtention de sériations céramiques systématique, site par site, sera également fondamentale pour mieux comprendre l'évolution et la diffusion des styles à l'intérieur des deux grandes régions mochica. Les études stylistiques devront être accompagnées d'analyses technologiques (voir Druc 2009 ; Ramon 2008, 2013) au sein de chaque site et vallée. Dans cette optique, une étude de cas sur les traditions technologiques du site de San José de Moro (vallée de Jequetepeque) est actuellement en cours au sein de l'IRAMATCRP2A de Bordeaux. Les résultats de cette recherche serviront à mieux définir les traits technologiques locaux dans les processus de production potière : elle permettra principalement de reconstruire les chaînes opératoires céramiques aux périodes Mochica tardive et transitionnelle. *

* Manuscrit reçu en mai 2014, accepté pour publication comme note de recherche en février 2015.

Nous tenons à remercier le programme IdEx Bordeaux (Initiative d'excellence de l'université de Bordeaux). Cette recherche a bénéficié d'une aide de l'État gérée par l'Agence 
nationale de la recherche au titre du programme Investissements d'avenir portant la référence ANR-10-LABX-52.

\section{Références citées}

ANDERs Martha

1981, « Investigation of State storage facilities in Pampa Grande, Peru », Journal of field archaeology, 8 (4), p. 391-404.

BAWDEN Garth

1982, « Galindo: a study in cultural transition during the Middle Horizon », in Michael E. Moseley et Kent, C. Day (éd.), Chan Chan: Andean desert city, University of New Mexico Press, Albuquerque, p. 285-320.

BelisLe Véronique

2008, « El Horizonte medio en el valle de Santa: continuidad y discontinuidad con los mochicas del Intermedio temprano », in Luis J. Castillo, Hélène Bernier, Gregory Lockard et Julio Rucabado (éd.), Arqueología mochica: nuevos enfoques. Actas del Primer congreso internacional de jóvenes investigadores de la cultura mochica (4-5 agosto 2004), Pontificia Universidad Católica del Perú/Instituto Francés de Estudios Andinos, UMIFRE 17-CNRS, Lima, p. 17-31.

BeyersdorfF Margot

2005, « Writing without words/Words without writing. The culture of the khipu », Latin American research review, 40 (3), p. 294-311.

BILLMAN Brian

2002, " Irrigation and the origins of the southern Moche state on the north coast of Peru », Latin American antiquity, 13 (4), p. 371-400.

Bird Broxton W., Mark B. Авbott, Mathias Vuille, Donald T. Rodbell, Michael Rosenmeier et Nathan D. StAnsell

2011a, « A 2,300-year-long annually resolved record of the South American summer monsoon from the Peruvian Andes ", Proceedings of the National academy of sciences of the United States of America, 108 (21), p. 8583-8588.

Bird Broxton W., Mark B. Аввотt, Donald T. Rodbell et Mathias Vuille

2011b, « Holocene tropical South American hydroclimate revealed from a decadally resolved lake sediment $\delta 180$ record », Earthand planetary science letters, 310, p. 192-202.

BRonk RAMSEY Christopher

2008, « Radiocarbon dating: revolution in understanding », Archaeometry, 50 (2), p. 249-275.

2014, « OxCal program, v. 4.2, Radiocarbon accelerator unit, University of Oxford, UK », http://c14.arch.ox.ac.uk/embed.php?File=oxcal.html, consulté le 10 octobre 2014.

Caracuta Valentina, Girolamo Fiorentino et Maria Clara Martinelli

2012, «Plants remains and AMS: dating climate change in the Aeolian Islands (Northeastern Sicily) during the 2nd millennium BC », Radiocarbon, 54 (3-4), p. 689-700. 


\section{Castillo Luis J.}

2000, « La presencia wari en San José de Moro », in Peter Kaulicke et William H. Isbell (éd.), Huari y Tiwanaku: modelos vs. evidencias, Boletín de arqueología PUCP IV, Pontificia Universidad Católica del Perú, Lima, p. 143-179.

2003, «Los últimos mochicas en Jequetepeque », in Santiago Uceda et Elías Mujica (éd.), Moche: hacia el final del milenio. Actas del segundo coloquio sobre la cultura moche (1-7 agosto 1999), Pontificia Universidad Católica del Perú/ Universidad Nacional de Trujillo, Lima, vol. 2, p. 65-123.

2010, « Moche politics in the Jequetepeque valley. A case for political opportunism », in Jeffrey Quilter et Luis J. Castillo (éd.), New perspectives on the Moche political organization, Dumbarton Oaks Research Library and Collection, Washington, p. 83-109.

2011, San José de Moro y la arqueología del valle de Jequetepeque, Fondo Editorial de la Pontificia Universidad Católica del Perú, Lima.

Castillo Luis J. et Christopher B. Donnan

1994, «Los mochicas del norte y los mochicas del sur: una perspectiva desde el Valle de Jequetepeque », in Krzysztof Makowski, Christopher B. Donnan et Ivan Amaro Bullon (éd.), Vicus, Banco del Crédito del Perú, Lima, p. 142-181.

Castillo Luis J. et Santiago Uceda

2008, « The Mochicas », in Helaine Silverman et William Isbell (éd.), Handbook of South American archaeology, Springer, New York, p. 707-729.

Chaparro Adolfo

2011, « Teo-iconology of sacrificial power among the Moche », AISTHESIS, 50, p. 72-91.

Chapdelaine Claude

2001, « The growing power of a Moche urban class », in Joanne Pillsbury (éd.), Moche art and archaeology in Ancient Peru, National Gallery of Art, Washington, p. 69-87.

2008, " Moche art style in Santa Valley: between being "à la mode" and developing a provincial identity », in Steve Bourget et Kimberly L. Jones (éd.), The art and archaeology of the Moche: an Ancient Andean society of the Peruvian north coast, University of Texas Press, Austin, p. 129-152.

2010, « Moche political organization in the Santa Valley: a case of direct rule through gradual control of the local population ", in Jeffrey Quilter et Luis J. Castillo (éd.), New perspectives on the Moche political organization, Dumbarton Oaks Research Library and Collection, Washington, p. 252-279.

2011, « Recent advances in Moche archaeology », Journal of archaeological research, 19 (2), p. 191-231.

Del Solar Nino

2011, La caractérisation archéométrique des céramiques précolombiennes du Pérou : une synthèse historique et méthodologique, mémoire de Master 1, université Michel-de-Montaigne Bordeaux 3.

Dillehay Tom D. et Alan L. Kolata

2004, « Long-term human response to uncertain environmental conditions in the Andes ", Proceedings of the National academy of sciences of the United States of America, 101 (12), p. 4325-4330. 
Dollwetzel Philipp

2012, Middle Horizon ceramic pigments. A meta-analysis of previous research on Peruvian ceramics in Bordeaux and Chemnitz, rapport de stage, Technische Universität, Chemnitz.

Donnan Christopher B.

1973, Moche occupation of the Santa Valley, Peru, University of California Press, Berkeley.

2007, Moche tombs at Dos Cabezas, Cotsen Institute of Archaeology, University of California, Los Angeles.

2011, « Moche substyles: keys to understanding Moche political organization », Boletín del Museo chileno de arte precolombino, 16 (1), p. 105-118.

Donnan Christopher B. et Donna McClelland

1997, « Moche burials at Pacatnamú », in Christopher B. Donnan et Guillermo Cock (éd.), The Pacatnamu papers. Volume 2, Fowler Museum of Cultural History, University of California, Los Angeles, p. 17-187.

DutTa Koushik

2008, « Marine 14C reservoir age and Suess effect in the Indian Ocean », e-Journal earth science India, 1 (3), p. 175-188.

DRUC Isabelle

2009, « Tradiciones alfareras, identidad social y el concepto de etnias tardías en Conchucos, Ancash, Perú », Bulletin de l'Institut français d'études andines, 38 (1), p. 87-106.

Finucane Brian C., J. Ernesto Valdez, Ismael Perez, Cirilo Vivanco, Lidio M. Valdez et Tamsin O'CONNELL

2007, «The end of empire: new radiocarbon dates from the Ayacucho Valley, Peru, and their implications for the collapse of the Wari state », Radiocarbon, 49 (2), p. 579-592.

Franco Régulo, César Galvez et Segundo Vasquez

2003, « Modelos, función y cronología de la Huaca Cao Viejo, complejo El Brujo », in Santiago Uceda et Elías Mujica (éd.), Moche: hacia el final del milenio. Actas del segundo coloquio sobre la cultura moche (1-7 agosto 1999), Pontificia Universidad Católica del Perú/Universidad Nacional de Trujillo, Lima, vol. 2, p. 125-177.

Grobman Alexander, Duccio Bonavia, Tom D. Dillehay, Dolores R. Piperno, José IRIARTE et Irene Holst

2012, " Preceramic maize from Paredones and Huaca Prieta, Peru », Proceedings of the National academy of sciences of the United States of America, 109 (5), p. 1755-1759, publié en ligne 17 janvier 2012 (doi : 10.1073/pnas.1120270109).

HAMmer Øyvind, David A. T. HARPER et Paul D. RyAN

2001, «PAST: Paleontological statistics software package for education and data analysis », Palaeontologia electronica, 4 (1), p. 9.

Haug Gerald H., Konrad A. Hughen, Daniel M. Sigman, Larry C. Peterson et Ursula RöHL 2001, «Southward migration of the intertropical convergence zone through the Holocene », Science, 293 (5533), p. 1304-1308. 
Hogg Alan G., Christopher Bronk Ramsey, Chris Turney et Jonathan Palmer 2009a, « Bayesian evaluation of the Southern hemisphere radiocarbon offset during the Holocene ", Radiocarbon, 51 (4), p. 1165-1176.

Hogg Alan G., Jonathan Palmer, Gretel Boswijk, Paula J. Reimer et David Brown $2009 \mathrm{~b}$, « Investigating the interhemispheric 14C offset in the 1st millenium AD and assessment of laboratory bias and calibration errors "), Radiocarbon, 51 (4), p. 1177-1186.

Hogg Alan G., Quan Hua, Paul G. Blackwell, Mu Niu, Caitlin E. Buck, Thomas P. Guilderson, Timothy J. Heaton, Jonathan G. Palmer, Paula J. Reimer, Ron W. Reimer, Christian S. M. Turney et Susan R. H. Zimmerman

2013, « Southern hemisphere calibration, 0-50,000 years cal BP », Radiocarbon, 55 (4), p. 1889-1903.

Johnson Frederick, Frank H. H. Roberts Jr., Robert F. Heizer, James B. Griffin, William S. WebB, William A. Ritchie, Helmut de Terra, Junius Bird, Hallam L. Movius Jr., Robert J. Braidwood, Thorkild Jacobsen, Richard A. Parker, Saul Weinberg, Richard Foster Flint, Edward S. Deevey Jr., Froelich Rainey et Donald Collier

1951, " Radiocarbon dating: a report on the program to aid in the development of the method of dating », Memoirs of the Society for American archaeology, 8, p. 1-65.

Kennett Douglas J., B. Lynn Ingram, John R. Southon et Karen Wise

2002, «Differences in 14C age between stratigraphically associated charcoal and marine shell from archaic period site of kilometer 4, southern Peru: old wood or old water? », Radiocarbon, 44 (1), p. 53-58.

Koons Michele et Bridget ALEX

2014, « Revised Moche chronology based on Bayesian models of reliable radiocarbon dates », Radiocarbon, 56 (3), p. 1039-1055.

LARCO Rafael

1944, « La escritura peruana sobre pallares », Relaciones de la sociedad argentina de antropología, 4, p. 57-76.

2001, Los mochicas, Museo arqueológico Rafael Larco Herrera, Lima, 2 vol.

Lechtman Heather, Antonieta ErLiJ et Edward Barry

1982, « New perspectives on Moche metallurgy: techniques of gilding copper at Loma Negra, northern Peru », American antiquity, 47 (1), p. 3-30.

LEÓN Elmo

2007, « Cronología de los fardos funerarios de Wari Kayan, Paracas Necrópolis », in Carmen Thays, Mary Frame, Elmo León, Johny Isla, Markus Reindel et Ann Peters (éd.), Hilos del pasado. El aporte francés al legado Paracas, Instituto nacional de Cultura, Lima, p. 33-47.

2011, « Los quipus y la calibración radiocarbónica », in Hugo Pereyra, Catherine Julien, Carmen Arellano and Gary Urton (éd.), Atando cabos, Ministerio de Cultura, Museo nacional de arqueología, antropología e historia del Perú, Lima, p. 227-237.

LOCKARD Gregory

2008, «A new view of Galindo: results of the Galindo archaeological project », in Luis J. Castillo, Hélène Bernier, Gregory Lockard et Julio Rucabado (éd.), Arqueología 
mochica: nuevos enfoques. Actas del Primer congreso internacional de jóvenes investigadores de la cultura mochica (4-5 agosto 2004), Pontificia Universidad Católica del Perú/Instituto Francés de Estudios Andinos, UMIFRE 17-CNRS, Lima, p. 275-293.

LOCKARD Gregory

2009, « The Occupational history of Galindo, Moche Valley, Peru », Latin American antiquity, 20 (2), p. 279-302.

MANGERUd Jan

1972, « Radiocarbon dating of marine shells, including a discussion of apparent age of recent shells from Norway », Boreas, 1, p. 143-172.

MANGerud Jan et Steinar Gulliksen

1974, « Apparent radiocarbon ages of recent marine shells from Norway, Spitsbergen, and Artic Canada », Quaternary research, 1 (2), p. 263-273.

Mangerud Jan, Stein Bondevik, Steinar Gulliksen, Anne Karin Hulfthammer et Tore HøISÆTER

2006, « Marine 14C reservoir ages for 19th century whales and molluscs from the north Atlantic », Quaternary science reviews, 25, p. 3228-3245.

MARSh Erik J.

2012, «A Bayesian re-assessment of the earliest radiocarbon dates from Tiwanaku, Bolivia », Radiocarbon, 54 (2), p. 203-218.

McCormac F. Gerry, Paula J. Reimer, Alan G. Hogg, Thomas F. G. Higham, Mike G. L. Baillie, Jonathan Palmer et Minze Stuiver

2002, « Calibration of the radiocarbon time scale for the southern hemisphere: AD 1850$950 »$, Radiocarbon, 44 (3), p. 641-651.

McCormac F. Gerry, Alan G. Hogg, Paul G. Blackwell, Caitlin E. Buck, Thomas F. G. Higham et Paula J. Reimer

2004, "SHCAL04 southern hemisphere calibration, 0-11.0 CAL KYR BP », Radiocarbon, 46 (3), p. 1087-1092.

Moscovich Viviana R.

2008, «El khipu como registro textil en el imperio inca: ¿herramienta de trabajo reutilizable o informe final? », Iberoamérica global, 1 (2), p. 59-93.

Moseley Michael E., Christopher B. Donnan et David D. Keefer

2008, « Convergent catastrophe and the demise of Dos Cabezas », in Steve Bourget et Kimberly L. Jones (éd.), The art and archaeology of the Moche: an Ancient Andean society of the Peruvian north coast, University of Texas Press, Austin, p. 81-91.

MoutARde Fanny

2008, « Los carbones hablan: un estudio del material antracológico de la Plataforma Uhle, Huaca de la Luna. Un acercamiento a la economía vegetal de la costa norte del Perú en la época mochica », in Luis J. Castillo, Hélène Bernier, Gregory Lockard et Julio Rucabado (éd.), Arqueología mochica: nuevos enfoques. Actas del Primer congreso internacional de jóvenes investigadores de la cultura 
mochica (4-5 agosto 2004), Pontificia Universidad Católica del Perú/Instituto Francés de Estudios Andinos, UMIFRE 17-CNRS, Lima, p. 295-305.

Nickel Daniela, Luis Jaime Castillo, Rémy Chapoulie, Nino Del Solar et Philipp Dollwetzel

2013, « Material analysis of colored pre-Columbian ceramic shards: identification of black pigments on ceramics of the Huari Viñaque, Mochica Polícromo and Cajamarca Costeño styles », in Andreas Hauptmann, Oliver Mecking et Michael Prange (éd.), Archäometrie und Denkmalpflege 2013. Jahrestagung an der Bauhaus-Universität Weimar (25-28 September 2013), Dt. Bergbau-Museum Bochum, (Metalla. Sonderheft, 6), Bochum, p. 148-153.

Ogburn Denise E.

2012, « Reconceiving the chronology of Inca imperial expansion », Radiocarbon, 54 (2), p. 219-237.

Ortlieb Luc, Gabriel Vargas et Jean-François Saliège

2011, « Marine radiocarbon reservoir effect along the northern Chile-southern Peru coast (12-24oS) throughout the Holocene », Quaternary research, 75 (1), p. 91-103.

Olsen Jesper, Jan Heinemeier, Harald LübKe, Friedrich Lüth et Thomas Terberger 2010, «Dietary habits and freshwater reservoir effects in bones from a Neolithic NE German cemetery », Radiocarbon, 52 (2), p. 635-644.

Petersen George

1980, «Aspectos generales de la costa peruana », in Mario Samamé Boggio (éd.), El Perú minero. Volume III. Geología, Instituto Geológico Minero y Metalúrgico, Lima, p. 35-42.

QUiLTER Jeffrey

2002, « Moche politics, religion and warfare », Journal of world prehistory, 16 (2), p. 145-195.

RAMÓN Gabriel

2008, « Producción alfarera en Piura (Perú): estilos técnicos y diacronía », Bulletin de l'Institut français d'études andines, 37 (3), p. 477-509.

2013, « Las fuentes del estilo: distribución regional de canteras y técnicas alfareras en Conchucos (Ancash, Perú) », Bulletin de l'Institut français d'études andines, 42 (1), p. 49-90.

Reimer Paula J., Edouard Bard, Alex Bayliss, J. Warren Beck, Paul G. Blackwell, Christopher Bronk Ramsey, Caitlin E. Buck, Hai Cheng, R. Lawrence Edwards, Michael Friedrich, Pieter M. Grootes, Thomas P. Guilderson, Haflidi Haflidason, Irka Hajdas, Christine Hatté, Timothy J. Heaton, Dirk L. Hoffmann, Alan G. HogG, Konrad A. Hughen, K. Felix Kaiser, Bernd Kromer, Sturt W. Manning, Mu Niu, Ron W. Reimer, David A. Richards, E. Marian Scott, John R. Southon, Richard A. Staff, Christian S. M. Turney et Johannes van der Plicht

2013, « IntCal13 and Marine13 radiocarbon age calibration curves 0-50,000 years Cal BP », Radiocarbon, 55 (4), p. 1869-1887. 
Rick Torben, René L. Vellanoweth et Jon M. ERLAndson

2005, "Radiocarbon dating and the "old shell" problem: direct dating of artifacts and cultural chronologies in coastal and other aquatic regions ", Journal of archaeological science, 32 (11), p. 1641-1648.

Roque Céline, Emmanuel Vartanian, Pierre Guibert, Max Schvoerer, Daniel Lévine, Walter Alva et Hogne JungneR

2002, « Recherche chronologique sur la culture mochica du Pérou: datation de la tombe du Prêtre de Sipán par thermoluminescence (TL) et par radiocarbone », Journal de la société des américanistes, 88, p. 227-243.

Rosas Marco

2007, « Nuevas perspectivas acerca del colapso moche en el bajo Jequetepeque. Resultados preliminares de la segunda campaña de investigación del proyecto arqueológico Cerro Chepén », Bulletin de l'Institut français d'études andines, 36 (2), p. 221-240.

Rowe John H.

1961, « Stratigraphy and seriation », American antiquity, 26 (3), p. 324-330.

Russell Glenn S., Banks I. LeONARD et Jesus Briceño

1998, « The Cerro Mayal workshop: addressing issues of craft specialization in Moche society ", in Izumi Shimada (éd.), Andean ceramics: technology, organization, and approaches, Museum Applied Science Center for Archaeology, University of Pennsylvania Museum of Archaeology and Anthropology (MASCA research papers in science and archaeology, suppl. to vol. 15), Philadelphia, p. 63-89.

Russell Glenn S. et Margareth JACKSON

2001, « Political economy and patronage at Cerro Mayal, Peru », in Joanne Pillsbury (éd.), Moche art and archaeology in Ancient Peru, National Gallery of Art, Washington, p. 159-175.

Sachs Julian P., Dirk Sachse, Rienk H. Smittenberg, Zhaohui Zhang, David S. Battisti et Stjepko Golubic

2009, « Southward movement of the Pacific intertropical convergence zone AD 1400$1850 »$, Nature geoscience, 2 (7), p. 519-525.

Scharf Andreas, Achim Bräuning, Wolfgang Kretschmer, Bertram Wegner et Frederique DARRAGON

2013, «14C AMS dating of wooden cores from historic buildings for archaeological and dendrochronological research in High Asia », Radiocarbon, 55 (2-3), p. $1358-1365$.

SHIMADA Izumi

1994, Pampa Grande and the Mochica culture, University of Texas Press, Austin.

Shimada Izumi, Crystal Barker S., Lonnie G. Thompson et Ellen Mosley-Thompson 1991, " Cultural impacts of severe droughts in the prehistoric Andes: application of a 1,500 year ice core », World archaeology, 22 (3), p. 247-270. 
Shimada Izumi et Adriana Maguiña

1994, « Nueva visión sobre la cultura gallinazo y su relación con la cultura moche », in Santiago Uceda et Elías Mujica (éd.), Moche: propuestas y perspectivas. Actas del Primer coloquio sobre la cultura moche (12-16 abril 1999), Universidad Nacional de la Libertad/Instituto Francés de Estudios Andinos (Travaux de 1'IFEA, 79)/ Asociación Peruana para el Fomento de las Ciencias Sociales, Trujillo, p. 31-58.

SKolmen Roger G.

1990, « Prosopis pallida (Humb. \& Bonpl., ex Willd.) H.B.K. Kiawe», in Russell M. Burns et Barbara H. Honkala (éd.), Silvics of North America. Vol. 2. Handbook, Forest Service, United States Department of Agriculture (Agriculture Handbook, 654), Washington, p. 583-586.

Stuiver Minze, Gordon. W. Pearson et Tom Braziunas

1986, « Radiocarbon age calibration of marine samples back to 9000 cal YR BP », Radiocarbon, 28 (2B), p. 980-1021.

Uceda Santiago, Claude Chapdelaine, Claude Chauchat et John W. Verano

2001, « Fechas radiocarbónicas para el complejo arqueológico Huacas del Sol y la Luna: una primera cronología del sitio », in Santiago Uceda (éd.), Informe del proyecto Huaca de la Luna 2001, Trujillo, p. 215-226.

ULm Sean

2006, "Short reports: Australian marine reservoir effects. A guide to $\Delta \mathrm{R}$ Values », Australian archaeology, 63, p. 57-60.

UNKEL Ingmar et Bernd KROMER

2009, « The clock in the corn cob: on the development of a chronology of the Paracas and Nasca Period based on radiocarbon dating », in Markus Reindel et Günther A. Wagner (éd.), New technologies for archaeology. Multidisciplinary investigations in Palpa and Nazca, Peru, Springer (Natural science in archaeology), Berlin, p. 231-244.

URTON Gary

2003, Signs of the Inka khipu: binary coding in the Andean knotted-string records, University of Texas Press, Austin.

VERANO John W.

2008, « Communality and diversity in Moche human sacrifice », in Steve Bourget et Kimberly L. Jones (éd.), The art and archaeology of the Moche: an Ancient Andean society of the Peruvian North coast, University of Texas Press, Austin, p. 195-213.

Weberbauer Augusto

1980, « Geografia física del Perú, orografía e hidrografía », in Mario Samamé Boggio (éd.), El Perú minero. Volume III. Geología, Instituto Geológico Minero y Metalúrgico, Lima, p. 12-34. 
